\title{
A Lyapunov-Krasovskii Functional Approach to Stability and Linear Feedback Synchronization Control for Nonlinear Multi- Agent Systems with Mixed Time Delays
}

\author{
A. Stephen $\left(\mathbb{D},{ }^{1}\right.$ R. Raja $\left(\mathbb{D},{ }^{2}\right.$ J. Alzabut $\left(\mathbb{D},{ }^{3,4}\right.$ Q. Zhu $\left(\mathbb{D},{ }^{5,6}\right.$ M. Niezabitowski ${ }^{\mathbb{D}},{ }^{7}$ \\ and C. P. $\operatorname{Lim}^{8}{ }^{8}$ \\ ${ }^{1}$ Department of Mathematics, Alagappa University, Karaikudi-630 004, India \\ ${ }^{2}$ Ramanujan Centre for Higher Mathematics, Alagappa University, Karaikudi-630 004, India \\ ${ }^{3}$ Department of Mathematics and General Sciences, Prince Sultan University, Riyadh 11586, Saudi Arabia \\ ${ }^{4}$ Group of Mathematics, Faculty of Engineering, Ostim Technical University, Ankara 06374, Turkey \\ ${ }^{5}$ School of Mathematics and Statistics, Hunan Normal University, Hunan 410 081, China \\ ${ }^{6}$ School of Information Science and Engineering, Chengdu University, Chengdu 610106, China \\ ${ }^{7}$ Faculty of Automatic Control Electronics and Computer Science, Department of Automatic Control and Robotics, \\ Silesian University of Technology, Akademicka 16, Gliwice 44-100, Poland \\ ${ }^{8}$ Institute for Intelligent System Research and Innovation, Deakin University, Australia
}

Correspondence should be addressed to Q. Zhu; zqx22@126.com

Received 23 December 2020; Revised 24 February 2021; Accepted 9 April 2021; Published 13 May 2021

Academic Editor: Alessandro Lo Schiavo

Copyright (c) 2021 A. Stephen et al. This is an open access article distributed under the Creative Commons Attribution License, which permits unrestricted use, distribution, and reproduction in any medium, provided the original work is properly cited.

This study focuses on mixed time delayed, both leaderless and leader-follower problems of nonlinear multi-agent systems. Here, we find the stability criteria for multi-agent systems (MASs) by utilizing a proposed lemma, the Lyapunov-Krasovskii functions, analytical techniques, Kronecker product, and some general specifications to obtain the asymptotic stability for the constructed MASs. Furthermore, the criteria to establish the synchronization of leader-follower multiagent systems with linear feedback controllers are discussed. Finally, we provide two numerical calculations along with the computational simulations to check the validity of the theoretical findings reported for both leaderless and leader-follower problem in this study.

\section{Introduction}

Multi-agent systems (MASs) have been well documented over the years as it is widely implemented in a variety of areas, including sensor networks, satellite sensors, unmanned-air-vehicle formulation, and joining multirobotics technology [1-3]. First, the idea of an agent as an artificial organism was introduced by a scientist named Holland [4]. The agent is a programmed process that includes a certain state and has the opportunity to communicate with other agents through the exchange of messages [5]. In 2015, Hu et al. [6] investigated a cooperative tracking for nonlinear multi-agent systems with a hybrid time-delayed protocol.
The related follower system is

$$
\dot{\mathcal{U}}_{i}(t)=\mathscr{A} \mathscr{U}_{i}(t)+\mathscr{B} \mathscr{K}_{i}(t)+\mathscr{D} \tilde{h}\left(t, \mathscr{U}_{i}(t)\right),
$$
where $\mathscr{U}_{i}(t) \in \mathscr{R}^{n}$ refers to the $i^{\text {th }}$ agent state. $\mathscr{K}_{i}(t) \in \mathscr{R}^{m}$ is the controller to be programmed. Let $\mathscr{A}, \mathscr{B}$, and $\mathscr{D}$ are the constant matrices.

The leader agent acts as a leader-follower problem command generator that generates the desired reference path and ignores the followers' knowledge. The information about the leader is accessed directly by a subset of the followers. The leader system is described as [6]

$$
\dot{u}_{0}(t)=\mathscr{A} U_{0}(t)+\mathscr{D} \tilde{h}\left(\mathscr{U}_{0}(t)\right),
$$

where $\mathscr{U}_{0}(t) \in \mathscr{R}^{n}$ denotes the leader state. 
The nonlinear systems evolved and grew rapidly under the distributed control of many nonlinear systems because they are practically seen everywhere. The results proposed under [7] are valid only for linear MASs dynamics. In [8], $\mathrm{H}$. Liang et al. derived neural network-based event-triggered adaptive control of nonaffine nonlinear multi-agent systems with dynamic uncertainties, in [9], Park et al. discussed betweenness centrality-based consensus protocol for second-order multi-agent systems with sampled data, and in [10], Park et al. proposed weighted consensus protocols design based on network centrality for multi-agent systems with sampled data. So far, in practice, intelligent agents are more likely to be manipulated by complex inherent nonlinear mechanics. The current literature includes only a few findings to explore the MASs problem with nonlinear dynamics. The authors discussed in $[11,12]$. Dynamic structures are often subjected to several disturbances in functional applications, such as communication delays. In general, the existence of delay is unavoidable, and this may lead to oscillation, divergence, instability, or other poor performances. If the considered multi-agent system does not attain the desired goal in the time period given and the signal communication process between the channels is very low, then there occurs some delay in getting the outputs. Then, the MASs is called the delayed multi-agent system. For example, in [13], Kwon et al. investigated the stability analysis of neural networks with interval time-varying delays via some new augmented Lyapunov-Krasovskii functional, in [14], W. Qin et al. discussed the impulsive observer-based consensus control for MASs with delayed protocol, in [15], the authors derived a composite feedback approach to stabilize a nonholonomic system with time-varying delays and nonlinear disturbances, in [16], Mobayen et al. investigated the robust global controller design for discrete time descriptor systems with multiple time-varying delays, in [17], Song et al. proposed a delay-dependent stability of nonlinear hybrid neutral stochastic differential equations with multiple delays, and in [18], the authors discussed closed centrality-based synchronization criteria for complex dynamical networks with interval time-varying coupling delays. The occurrence of communication delays is therefore essential for the study of the nonlinear MASs problem. The dynamics of MASs with time delays remain active based on the past shreds of data, and it has been a very hot topic of research among several scientific researchers [19]. Hu et al. derived a distributed containment control for nonlinear MASs with time delayed protocol. Also, the author's in [13] discussed about the Lyapunov stability for the developed neural networks with the inclusion of interval time-varying delays and some new augmented Lyapunov-Krasovskii functional.

Problems on different multiagents, according to our earlier literature survey many scholars, have spoken about the structure of a leader. For example, Jia et al. investigated the leader-follower nonlinear MASs and coupling delay in [20]. In many of the references on leader-follower problem [21-23], leaderless principles [24], the leaderless and leader-follower consensus by Meng et al. [24] with communication and input delays, the authors fails to study the stability and synchronization analysis of nonlinear MASs with mixed delays, and it is facing a challenge in the field concerned.

In 2016, He et al. [25] analyzed the leader-follower consensus of nonlinear multiagent systems. In those studies, the authors considered a leader-follower multiagent system with one leader and $N$ followers.

Accordingly, the leader system was framed as

$$
\dot{\mathcal{U}}_{0}(t)=\mathscr{A} \mathcal{U}_{0}(t)+\mathscr{B} \tilde{h}\left(\mathcal{U}_{0}(t)\right),
$$

where $\mathscr{U}_{0}(t) \in \mathscr{R}^{n}$ denotes the state of the leader; $\widetilde{h}\left(\mathscr{U}_{0}(t)\right)=\left(\widetilde{h}_{1}\left(\mathscr{U}_{0}(t)\right), \widetilde{h}_{2}\left(\mathscr{U}_{0}(t)\right), \ldots, \widetilde{h}_{n}\left(\mathscr{U}_{0}(t)\right)\right)^{T}$ is defined as a nonlinear function. Let $\mathscr{A}$ and $\mathscr{B}$ are the constant matrices.

Furthermore, the follower system can be described as follows:

$$
\dot{\mathcal{U}}_{i}(t)=\mathscr{A} \mathcal{U}_{i}(t)+\mathscr{B} \tilde{h}\left(\mathcal{U}_{i}(t)\right)+\mathscr{K}_{i}(t),
$$

where $\mathscr{U}_{i}(t) \in \mathscr{R}^{n}$ refers to the $i^{\text {th }}$ agent state, and $\mathscr{K}_{i}(t)$ is the controller designed.

It is noteworthy to consider time delays in the model and study their stability and synchronization, since it is unavoidable. Delays are of two types, one is discrete and the other one is distributed. Nowadays, distributed time delays have received more research attention, since each network generally has a spatial character because there are parallel paths of several axon sizes and length. Note that the findings related to the initial research of the concept of multiagent systems with distributed delays are very few [26]. Although it can be seen that the distributed time delays in the transmission of signals delivered at a given time, it may also be immediate at some point to coordinate the distributed delay. For a given period of time, the limited distributed delay should be used to compare the current behavior of the state with the distant past, which has less impact. For example, many research works are related to the stability of mixed delay $[13,27]$.

In recent years, some interest and excellent achievements have been achieved to address the stability problem of a system with delays $[28,29]$. In [30], Kong et al. derived a new fixed-time stability lemmas and applications to discontinuous fuzzy inertial neural networks. In multi-agent systems, every agent exchanges information with its neighbors' agents to achieve a goal. Current literature centered primarily on the issue of multi-agent systems with integrative, first-order, second-order, and higher-order dynamics involving linear or nonlinear behaviors. This study analyses the nonlinear multi-agent systems of discrete and distributed delays.

On the other side, however, the leader-follower topic in multi-agent systems has been considered. For example, $\mathrm{Li}$ and Zhou proposed an impulsive coordination of nonlinear MASs with multiple leaders and stochastic disturbances in [31], and Zhou et al. investigated the leaderfollower exponential consensus of general linear MASs via event-triggered control [7]. Synchronization means that the couple of two dynamic systems (leader and controller of the follower) can achieve the same time with the same partial state. In the above comparisons, the proposed results showed 
that the actions of the follower system had an effect on the leader systems because the leader system did not depend on the follower systems. In other words, the leader system always transmits the communication signal that gives rise to the follower through channels, and this signal results in coordination with the leader. In [32], Ali et al. addressed the synchronization analysis for stochastic T-S fuzzy complex networks with Markovian jumping parameters and mixed time-varying delays via impulsive control, in [33], Kong et al. proposed a fixed-time synchronization analysis for discontinuous fuzzy inertial neural networks with parameter uncertainties, in [34], Kong and Zhu derived a new fixedtime synchronization control of discontinuous inertial neural networks via the indefinite Lyapunov-Krasovskii functional method. Synchronization problems have been studied in $[35,36]$. Generally speaking, synchronization can be of several types. Many researchers have discussed lag synchronization, projective lag synchronization [37], adaptive lag synchronization, antisynchronization, adaptive synchronization [38], and so on. To this evidence, the author's in [12] discussed the exponential synchronization/ consensus for nonlinear MASs with communication and input delays via a hybrid controller.

To achieve a multi-agent systems target, various control schemes such as impulsive control $[39,40]$, pinning control [41, 42], and adaptive control [41, 43], in [44], He et al. derived boundary vibration control of variable length crane systems in two-dimensional space with output constraints, in [45], Saravanakumar et al. derived a finite-time sampled data control of the switched stochastic model with nondeterministic actuator faults and saturation nonlinearity, and in [46], He et al. addressed an unified iterative learning control for flexible structures with input constraints. In [47], the robust finite-time composite nonlinear feedback control for synchronization of uncertain chaotic systems with nonlinearity and time delay has been implemented. To the best of our knowledge, in MASs, both the analysis of stability and synchronization are not yet tackled in the existing literature; hence, this situation sparks a further investigation of multi-agent systems for stability and synchronization.

Motivated by these results, this study focuses on the analysis of leaderless and leader-follower problems by a linear feedback control for a class of nonlinear multiagent systems. The key contributions of this study can be summarized as follows:
(1) In this study, we solve the asymptotic stability and synchronization of multi-agent systems with mixed time delays

(2) The main section deals with the asymptotic stability of multiagent systems with mixed delays, linear matrix inequality (LMI), Lyapunov-Krasovskii functions, and Kronecker product

(3) A novel linear feedback controller is proposed to realize the synchronization

(4) Finally, we provide two computational simulations to check the validity of the theoretical findings that are suggested

The rest of this study is organized as follows. In Section 2, the system description and some preliminaries are involved. In Section 3, the asymptotic stability of MASs is discussed. In Section 4, a linear feedback control is designed, and several sufficient conditions for asymptotic synchronization are obtained. In Section 5, two numerical examples are given to illustrate the feasibility of the proposed theoretical results. Finally, conclusions are drawn in Section 6.

Notation. Throughout the entire study, $\mathscr{R}^{n}$ and $\mathscr{R}^{n \times n}$ represent the $n$-dimensional Euclidean space as well as the set of all $n \times n$ real matrices. $\|$.$\| refers to the Euclidean norm.$ $\otimes$ refers to the Kronecker product. In a symmetric matrix, the notation $\star$ always denotes the symmetric component.

\section{Problem Statement and Preliminaries}

Let $G=(\nu, \varepsilon, \mathscr{W})$ be the graph of the interconnection between $N$ agents with the node set $\nu=\left\{\mathfrak{b}_{1}, \mathfrak{b}_{2}, \ldots, \mathfrak{b}_{N}\right\}$ and the set of directed edges $\varepsilon \subseteq\{\nu \times \nu\}$. If $\left(\mathfrak{b}_{i}, \mathfrak{b}_{k}\right) \in \mathcal{E}$, then there is an edge from the $\mathfrak{v}_{k}$ node to the $\mathfrak{b}_{i}$ node, meaning that $\mathfrak{v}_{k}$ is a $\mathfrak{b}_{i}$ neighbor, and $\mathscr{W}=\left[\mathfrak{a}_{i k}\right]_{N \times N}$ is the weighted adjacency matrix of graph $G . \mathfrak{a}_{i k}>0$ if and only if there is an edge from agent $\mathfrak{b}_{k}$ to node $\mathfrak{v}_{i}$. The set of neighbors of agent $i$ is denoted by $\mathcal{N}_{i}=\left\{\mathfrak{b}_{k} \in v:\left(\mathfrak{b}_{i}, \mathfrak{b}_{k} \in \varepsilon\right)\right\}$. A directed path from agent $\mathfrak{b}_{i}$ to agent $\mathfrak{b}_{k}$ is a sequence of edges $\left(\mathfrak{v}_{i}, \mathfrak{v}_{l_{1}}\right),\left(\mathfrak{v}_{l_{1}}, \mathfrak{v}_{l_{2}}\right), \ldots,\left(\mathfrak{v}_{l_{m}}, \mathfrak{v}_{k}\right)$. If each node has a directional path to each other, it is strongly related to the directed graph. The Laplacian matrix $\mathscr{L}=\left(\mathfrak{l}_{i k}\right)_{N \times N}$ of the graph $G$ is defined by $\mathfrak{l}_{i k}=-\mathfrak{a}_{i k}, i \neq j, \mathfrak{l}_{i k}=\sum_{k=1, k \neq i}^{N} \mathfrak{l}_{i k}(i, k=1,2,3, \ldots, N)$.

Consider the following multi-agent systems (MASs) with consisting $N$ agents as follows:

$$
\left\{\begin{array}{l}
\dot{U}_{i}(t)=\mathscr{A} \mathscr{U}_{i}(t)+\mathscr{B} \tilde{h}\left(\mathcal{U}_{i}(t)\right)+\mathscr{C} \widetilde{h}\left(\mathcal{U}_{i}(t-m(t))\right)+\mathscr{D} \int_{t-\varrho(t)}^{t} \tilde{h}\left(\mathcal{U}_{i}(\sigma)\right) d \sigma+\sum_{k=1}^{N} \mathfrak{a}_{i k}\left(\mathscr{U}_{k}(t)\right), \\
\mathcal{U}_{i}(t)=\mathscr{U}_{i}^{0}(t), \quad t \in[-\hat{\eta}, 0],
\end{array}\right.
$$

where $i=1,2,3, \ldots, N ; \mathscr{U}_{i}(t) \in \mathscr{R}^{n}$ is the $i^{\text {th }}$ agent state; $\widetilde{h}: \mathscr{R}^{n} \longrightarrow \mathscr{R}^{n}$ describes the intrinsic dynamics of each agent. Let $\mathscr{A}, \mathscr{B}, \mathscr{C}$, and $\mathscr{D}$ are the known matrices of $\mathscr{R}^{n \times n}$. $m(t)$ is the discrete delay, and $\varrho(t)$ is the distributed delay that satisfies $0 \leq m_{1} \leq m(t) \leq m_{2}, \dot{m}(t) \leq \lambda$, and $0<\varrho(t) \leq \varrho$, where $m_{1}, m_{2}, \lambda$, and $\varrho$ are the constants and $\hat{\eta}=$ $\max \left\{m_{1}, m_{2}, \varrho\right\}$.

The compact form of the MASs (5) is 


$$
\left\{\begin{array}{l}
\dot{U}(t)=(-\mathscr{L} \otimes \mathscr{A}) \mathscr{U}(t)+(\mathscr{I} \otimes \mathscr{B}) \widetilde{h}(\mathscr{U}(t))+(\mathscr{I} \otimes \mathscr{C}) \widetilde{h}(\mathscr{U}(t-m(t)))+(\mathscr{I} \otimes \mathscr{D}) \int_{\mathrm{t}-\varrho(t)}^{t} \tilde{h}(\mathscr{U}(\sigma)) \mathrm{d} \sigma \\
\mathcal{U}(t)=\mathscr{U}^{0}(t), \quad t \in[-\widehat{\eta}, 0]
\end{array}\right.
$$

where $\mathscr{U}(t)=\left[\mathcal{U}_{1}, \mathscr{U}_{2}, \ldots, \mathscr{U}_{N}\right]^{T}$ denotes the state vector, and $\hat{\eta}=\max \left\{m_{1}, m_{2}, \varrho\right\}$.

Lemma 1 (See [48]). The following inequality holds to any real matrices $\mathscr{X}, \mathcal{Y}$, constant $\Theta>0$, and any positive matrix $M$ :

$$
2 \mathscr{X}^{T} \mathcal{Y} \leq \omega \mathscr{X}^{T} M \mathscr{X}+\omega^{-1} \mathscr{Y}^{T} M^{-1} \mathcal{Y}
$$

Lemma 2 (See [13]). For any positive definite matrix $M \in \mathscr{R}^{n \times n}, \quad$ scalars $\quad \beta>\alpha>0$, vector function $\mathcal{U}:[\alpha, \beta] \longrightarrow \mathscr{R}^{n}$, the following inequality holds that the integrations concerned are well defined:

$$
-\alpha \int_{\beta}^{\alpha} \mathscr{U}^{T}(\sigma) M \mathscr{U}(\sigma) \mathrm{d} \sigma \leq-\left(\int_{\beta}^{\alpha} \mathcal{U}(\sigma) \mathrm{d} \sigma\right)^{T} M\left(\int_{\beta}^{\alpha} \mathcal{U}(\sigma) \mathrm{d} \sigma\right) .
$$

Lemma 3 (See [25]). One has matrices $\mathscr{A}, \mathscr{B}, \mathscr{C}$, and $\mathscr{D}$ with suitable dimensions.
(i) $(\mathscr{A} \otimes \mathscr{B})^{T}=\mathscr{A}^{T} \otimes \mathscr{B}^{T}$
(ii) $(\mathscr{A}+\mathscr{B}) \otimes \mathscr{C}=(\mathscr{A} \otimes \mathscr{C})+(\mathscr{B} \otimes \mathscr{C})$
(iii) $(\mathscr{A} \otimes \mathscr{B})(\mathscr{C} \otimes \mathscr{D})=\mathscr{A} \mathscr{C} \otimes \mathscr{B} \mathscr{D}$

Lemma 4 (See [49]). Linear matrix inequality (LMI) is given as follows:

$$
\left(\begin{array}{cc}
\widehat{Q}_{11} & \widehat{Q}_{12} \\
\widehat{Q}_{12}^{T} & \widehat{Q}_{22}
\end{array}\right)<0
$$

where $\widehat{Q}_{11}=\widehat{Q}_{11}^{T}$ and $\widehat{Q}_{22}=\widehat{Q}_{22}^{T}$ is equivalent to the following conditions:

$$
\begin{aligned}
& \text { (1) } \widehat{Q}_{11}<0, \widehat{Q}_{22}-\widehat{Q}_{12}^{T} \widehat{Q}_{11}^{-1} \widehat{Q}_{12}<0 \\
& \text { (2) } \widehat{Q}_{22}<0, \widehat{Q}_{11}-\widehat{Q}_{12} \widehat{Q}_{22}^{-1} \widehat{Q}_{12}^{T}<0
\end{aligned}
$$

Definition 1 (See $[49,50]$ ). An equilibrium point of the multi-agent systems (5) is a constant vector $\mathscr{U}^{*} \in \mathscr{R}^{n}$ which satisfies the following equation:

$$
0=(\mathscr{A}-\mathscr{L}) \mathcal{U}_{i}^{*}+\mathscr{B} \widetilde{h}\left(\mathcal{U}_{i}^{*}\right)+\mathscr{C} \tilde{h}\left(\mathcal{U}_{i}^{*}\right)+\mathscr{D} \tilde{h}\left(\mathcal{U}_{i}^{*}\right) .
$$

Definition 2 (See [23]). The equilibrium point of the multiagent systems (14) is said to be asymptotically stable if and only if for any initial conditions $\mathscr{P}_{i}(\sigma)$ on $-\widehat{\eta} \leq \sigma \leq 0$,

$$
\begin{array}{r}
\lim _{\mathrm{t} \longrightarrow \infty}\left\|\mathscr{P}_{i}(t)\right\|^{2}=\lim _{\mathrm{t} \longrightarrow \infty}\left\|\mathcal{U}_{i}(t)-\mathcal{U}^{*}\right\|^{2}=0, \\
\forall i=1,2, \ldots, N .
\end{array}
$$

Definition 3. (See [23]). The error of the multi-agent systems (48) is said to be asymptotic synchronization if and only if for any initial conditions $\mathcal{S}_{i}(\sigma)$ on $-\widehat{\eta} \leq \sigma \leq 0$,

$$
\begin{array}{r}
\lim _{t \rightarrow \infty}\left\|\mathcal{S}_{i}(t)\right\|^{2}=\lim _{t \longrightarrow \infty}\left\|\mathcal{U}_{i}(t)-\mathscr{U}_{0}(t)\right\|^{2}=0, \\
\forall i=1,2, \ldots, N .
\end{array}
$$

Assumption 1. There exist constant matrix $\mathscr{M} \in \mathscr{R}^{n \times n}$, such that for $\forall \mathfrak{U}, \mathfrak{V} \in \mathscr{R}$, satisfying

$$
\|\tilde{h}(\mathfrak{U})-\tilde{h}(\mathfrak{V})\| \leq\|\mathscr{M}(\mathfrak{U}-\mathfrak{V})\| .
$$

Remark 1. The multi-agent systems (6) is more advanced than the existing works in the available studies [20, 21, 31]. In [20], Jia et al. derived a leader-following of nonlinear agents with the switching connective network and coupling delay, in [31], Li et al. addressed an impulsive coordination of nonlinear multi-agent systems with multiple leaders and stochastic disturbance, and in [21], Ni and Cheng discussed a leader-follower consensus of multi-agent systems under fixed and switching topologies. While modeling a multiagent systems, the existence of both discrete and distributed delay is unavoidable, and sometimes, it leads to a worse dynamical behavior. The authors in [26] considered mixed delay terms as a constant one. But in our proposed study, we consider the mixed delay as time-varying. Hence, this shows that the proposed model improves the other existing ones in the known source of literature.

\section{Main Results}

In the following section, we introduce the discrete and distributed time delays into the multi-agent systems and to check the system stability.

3.1. Stability Criteria. In this subsection, we will show the asymptotic stability of the multi-agent systems.

In order to achieve the stability criteria, first, we shift the point of equilibrium for the considered multi-agent systems (1) to the origin. Then, the transformation is denoted by $\mathscr{P}_{i}(t)=\mathscr{U}_{i}(t)-\mathscr{U}^{*}$.

Hence, the system can be modified as follows: 


$$
\left\{\begin{array}{l}
\dot{\mathscr{P}}_{i}(t)=\mathscr{A} \mathscr{P}_{i}(t)+\mathscr{B} \tilde{g}\left(\mathscr{P}_{i}(t)\right)+\mathscr{C} \widetilde{\mathcal{g}}\left(\mathscr{P}_{i}((t-m(t)))\right)+\mathscr{D} \int_{\mathrm{t}-\varrho(t)}^{t} \tilde{\mathfrak{g}}\left(\mathscr{P}_{i}(\sigma)\right) \mathrm{d} \sigma-\mathscr{L} \mathscr{P}_{i}(t), \\
\mathscr{P}_{i}(t)=\mathscr{P}_{i}^{0}(t), \quad \mathrm{t} \in[-\widehat{\eta}, 0],
\end{array}\right.
$$

where $g\left(\mathscr{P}_{i}(t)\right)=\widetilde{h}\left(\mathcal{U}_{i}(t)+\mathscr{U}^{*}\right)-\widetilde{h}\left(\mathcal{U}^{*}\right)$, where $i=$ $1,2,3, \ldots, N ; \mathscr{P}_{i}(t) \in \mathscr{R}^{n}$ is the $i^{\text {th }}$ agent state; $\tilde{g}: \mathscr{R}^{n} \longrightarrow \mathscr{R}^{n}$ describing the inherent dynamics of each agent.

The compact form of the above system is given as follows:

$$
\begin{aligned}
\dot{\mathscr{P}}(t)= & (-\mathscr{L} \otimes \mathscr{A}) \mathscr{P}(t)+(\mathscr{I} \otimes \mathscr{B}) \tilde{g}(\mathscr{P}(t)) \\
& +(\mathscr{I} \otimes \mathscr{C}) \tilde{g}(\mathscr{P}(t-m(t))) \\
& +(\mathscr{I} \otimes \mathscr{D}) \int_{\mathrm{t}-\varrho(t)}^{t} \tilde{g}(\mathscr{P}(\sigma)) \mathrm{d} \sigma .
\end{aligned}
$$

$$
\Delta=\left[\begin{array}{ccccccccc}
\bar{\Omega}_{11} & 0 & 0 & 0 & 0 & 0 & 0 & 0 & 0 \\
\star & \bar{\Omega}_{22} & 0 & 0 & 0 & 0 & 0 & 0 & 0 \\
\star & \star & \bar{\Omega}_{33} & 0 & 0 & 0 & 0 & 0 & 0 \\
\star & \star & \star & \bar{\Omega}_{44} & 0 & 0 & 0 & 0 & 0 \\
\star & \star & \star & \star & \bar{\Omega}_{55} & 0 & 0 & 0 & 0 \\
\star & \star & \star & \star & \star & \bar{\Omega}_{66} & 0 & 0 & 0 \\
\star & \star & \star & \star & \star & \star & \bar{\Omega}_{77} & 0 & 0 \\
\star & \star & \star & \star & \star & \star & \star & \bar{\Omega}_{88} & 0 \\
\star & \star & \star & \star & \star & \star & \star & \star & \bar{\Omega}_{99}
\end{array}\right]<0
$$

Theorem 1. The system (15) can be achieved asymptotically stable for given positive constants $m_{1}, m_{2}, \varrho$, and $\lambda$ if there exists positive scalars $\omega$ and $\omega_{\mathfrak{K}}$, where $\mathfrak{\Omega}=1,2, \ldots, 11$ and positive matrices $\mathbb{Q}_{\mathfrak{K}} \in \mathscr{R}^{n \times n}$, where $\mathfrak{\Re}=1,2, \ldots, 8$, and any matrix $\mathscr{H}$ is holding the following inequality:

$$
\begin{aligned}
& \bar{\Omega}_{11}=-2\left(\mathscr{L} \otimes Q_{1} \mathscr{A}\right)+m_{1}\left(\mathscr{I} \otimes Q_{2}\right)+m_{2}\left(\mathscr{I} \otimes Q_{3}\right)+\left(\mathscr{I} \otimes Q_{6}\right)+\left(\mathscr{I} \otimes Q_{7}\right)+\left(\mathscr{I} \otimes \mathscr{M}^{T} \mathscr{M}\right) \\
& +\varpi_{1}\left(\mathscr{I} \otimes \mathbb{Q}_{1} \mathscr{B}\right)\left(\mathscr{I} \otimes Q_{1} \mathscr{B}\right)^{T}+\omega_{2}\left(\mathscr{I} \otimes Q_{1} \mathscr{C}\right)\left(\mathscr{I} \otimes Q_{1} \mathscr{C}\right)^{T}+\omega_{3}\left(\mathscr{I} \otimes Q_{1} \mathscr{D}\right)\left(\mathscr{I} \otimes Q_{1} \mathscr{D}\right)^{T}+\Phi_{4}\left(\mathscr{I} \otimes \mathscr{H}(\mathscr{I} \otimes \mathscr{H})^{T}\right. \\
& -\omega_{5}(\mathscr{I} \otimes \mathscr{H} \mathscr{B})(\mathscr{I} \otimes \mathscr{H} \mathscr{B})^{T}-\omega_{6}(\mathscr{I} \otimes \mathscr{H} \mathscr{C})(\mathscr{I} \otimes \mathscr{H} \mathscr{C})^{T}-\omega_{7}(\mathscr{I} \otimes \mathscr{H} \mathscr{D})(\mathscr{I} \otimes \mathscr{H} \mathscr{D})^{T}+\omega_{8}^{-1}-2(\mathscr{I} \otimes \mathscr{H} \mathscr{A}) \text {, } \\
& \bar{\Omega}_{22}=-(1-\lambda)\left(\mathscr{I} \otimes Q_{6}\right) \text {, } \\
& \bar{\Omega}_{33}=-m_{1}\left(\mathscr{I} \otimes Q_{2}\right)+\left(\mathscr{I} \otimes Q_{4}\right) \text {, } \\
& \bar{\Omega}_{44}=-m_{2}\left(\mathscr{I} \otimes Q_{3}\right)-\left(\mathscr{I} \otimes Q_{4}\right) \text {, } \\
& \bar{\Omega}_{55}=-2 \omega(\mathscr{I} \otimes \mathscr{H})-\omega_{8} \omega^{2}(\mathscr{L} \otimes \mathscr{H} \mathscr{A})(\mathscr{L} \otimes \mathscr{H} \mathscr{A})^{T}+\omega_{9} \omega^{2}(\mathscr{I} \otimes \mathscr{H} \mathscr{B})(\mathscr{I} \otimes \mathscr{H} \mathscr{B})^{T}+\varpi_{4}^{-1} \\
& +\omega_{10} \omega^{2}(\mathscr{I} \otimes \mathscr{H} \mathscr{C})(\mathscr{I} \otimes \mathscr{H} \mathscr{C})^{T}+\omega_{11} \omega^{2}(\mathscr{I} \otimes \mathscr{H} \mathscr{D})(\mathscr{I} \otimes \mathscr{H} \mathscr{D})^{T}, \\
& \bar{\Omega}_{66}=-1+\left(\mathscr{I} \otimes Q_{5}\right)+\varrho^{2}\left(\mathscr{I} \otimes Q_{8}\right)+\omega_{1}^{-1}-\omega_{5}^{-1}+\omega_{9}^{-1}, \\
& \bar{\Omega}_{77}=-(1-\lambda)\left(\mathscr{I} \otimes Q_{5}\right)+\omega_{2}^{-1}-\varpi_{6}^{-1}+\varpi_{10}^{-1} \text {, } \\
& \bar{\Omega}_{88}=-\left(\mathscr{I} \otimes Q_{7}\right) \text {, } \\
& \bar{\Omega}_{99}=-\left(\mathscr{I} \otimes Q_{8}\right)+\omega_{3}^{-1}+\omega_{11}^{-1}-\varpi_{7}^{-1} \text {. }
\end{aligned}
$$


Proof. Construct the Lyapunov-Krasovskii function as

$$
\mathscr{V}(t)=\sum_{i=1}^{8} \mathscr{V}_{i}(t)
$$

where $\quad \mathscr{V}_{1}(t)=\mathscr{P}^{T}(t)\left(\mathscr{I} \otimes Q_{1}\right) \mathscr{P}(t)$,

$$
\begin{aligned}
& \mathscr{V}_{2}(t)=m_{1} \int_{\mathrm{t}-m_{1}}^{\mathrm{t}} \mathscr{P}^{T}(\sigma)\left(\mathscr{I} \otimes \mathscr{Q}_{2}\right) \mathscr{P}(\sigma) \mathrm{d} \sigma, \\
& \mathscr{V}_{3}(t)=m_{2} \int_{\mathrm{t}-m_{2}}^{\mathrm{t}} \mathscr{P}^{T}(\sigma)\left(\mathscr{I} \otimes \mathscr{Q}_{3}\right) \mathscr{P}(\sigma) \mathrm{d} \sigma,
\end{aligned}
$$$$
\mathscr{V}_{4}(t)=\int_{t-m_{2}}^{t-m_{1}} \mathscr{P}^{T}(\sigma)\left(\mathscr{I} \otimes \mathscr{Q}_{4}\right) \mathscr{P}(\sigma) \mathrm{d} \sigma
$$

$$
\mathscr{V}_{5}(t)=\int_{t-m(t)}^{t} \tilde{\mathfrak{g}}^{T}(\mathscr{P}(\sigma))\left(\mathscr{I} \otimes Q_{5}\right) \widetilde{g}(\mathscr{P}(\sigma)) \mathrm{d} \sigma,
$$$$
\mathscr{V}_{6}(t)=\int_{t-m(t)}^{t} \mathscr{P}^{T}(\sigma)\left(\mathscr{I} \otimes Q_{6}\right) \mathscr{P}(\sigma) \mathrm{d} \sigma
$$$$
\mathscr{V}_{7}(t)=\int_{t-\varrho(t)}^{t} \mathscr{P}^{T}(\sigma)\left(\mathscr{I} \otimes Q_{7}\right) \mathscr{P}(\sigma) \mathrm{d} \sigma,
$$$$
\mathscr{V}_{8}(t)=\varrho \int_{-\varrho(t)}^{0} \int_{\mathrm{t}+\theta}^{\mathrm{t}} \tilde{g}^{T}(\mathscr{P}(\sigma))\left(\mathscr{I} \otimes Q_{8}\right) \widetilde{g}(\mathscr{P}(\sigma)) \mathrm{d} \sigma \mathrm{d} \theta .
$$

The time derivative of (18) will then be

$$
\begin{aligned}
& \dot{\mathscr{V}}_{1}(t)=2 \mathscr{P}^{T}(t)\left(\mathscr{I} \otimes Q_{1}\right) \dot{\mathscr{P}}(t) \\
& \dot{\mathscr{V}}_{1}(t)=2 \mathscr{P}^{T}(t)\left(\mathscr{I} \otimes Q_{1}\right)[-(\mathscr{L} \otimes \mathscr{A}) \mathscr{P}(t)+(\mathscr{I} \otimes \mathscr{B}) \widetilde{g}(\mathscr{P}(t))+(\mathscr{I} \otimes \mathscr{C}) \tilde{g}(\mathscr{P}(t-m(t))) \\
& \left.+(\mathscr{I} \otimes \mathscr{D}) \int_{t-\varrho(t)}^{t} \tilde{g}(\mathscr{P}(\sigma)) \mathrm{d} \sigma\right] \\
& =2 \mathscr{P}^{T}(t)\left(-\mathscr{L} \otimes \mathscr{Q}_{1} \mathscr{A}\right) \mathscr{P}(t)+2 \mathscr{P}^{T}(t)\left(\mathscr{I} \otimes Q_{1} \mathscr{B}\right) \widetilde{g}(\mathscr{P}(t))+2 \mathscr{P}^{T}(t)\left(\mathscr{I} \otimes Q_{1} \mathscr{C}\right) \widetilde{g}(\mathscr{P}(t-m(t))) \\
& +2 \mathscr{P}^{T}(t)\left(\mathscr{I} \otimes Q_{1} \mathscr{D}\right) \int_{t-\varrho(t)}^{t} \tilde{g}(\mathscr{P}(\sigma)) \mathrm{d} \sigma \\
& \dot{\mathscr{V}}_{2}(t)=m_{1} \mathscr{P}^{T}(t)\left(\mathscr{I} \otimes \mathcal{Q}_{2}\right) \mathscr{P}(t)-m_{1} \mathscr{P}^{T}\left(t-m_{1}\right)\left(\mathscr{I} \otimes \mathscr{Q}_{2}\right) \mathscr{P}\left(t-m_{1}\right) \\
& \dot{\mathscr{V}}_{3}(t)=m_{2} \mathscr{P}^{T}(t)\left(\mathscr{I} \otimes \mathcal{Q}_{3}\right) \mathscr{P}(t)-m_{2} \mathscr{P}^{T}\left(t-m_{2}\right)\left(\mathscr{I} \otimes \mathscr{Q}_{3}\right) \mathscr{P}\left(t-m_{2}\right) \\
& \dot{\mathscr{V}}_{4}(t)=\mathscr{P}^{T}\left(t-m_{1}\right)\left(\mathscr{I} \otimes \mathbb{Q}_{4}\right) \mathscr{P}\left(t-m_{1}\right)-\mathscr{P}^{T}\left(t-m_{2}\right)\left(\mathscr{I} \otimes \mathbb{Q}_{4}\right) \mathscr{P}\left(t-m_{2}\right) \\
& \dot{\mathscr{V}}_{5}(t)=\tilde{g}^{T}(\mathscr{P}(t))\left(\mathscr{I} \otimes Q_{5}\right) \tilde{g}(\mathscr{P}(t))-(1-\dot{m}(t)) \tilde{g}^{T}(\mathscr{P}(t))\left(\mathscr{I} \otimes \mathscr{Q}_{5}\right) \widetilde{g}(\mathscr{P}(t)), \\
& \leq \tilde{g}^{T}(\mathscr{P}(t))\left(\mathscr{I} \otimes Q_{5}\right) \widetilde{g}(\mathscr{P}(t))-(1-\lambda) \tilde{g}^{T}(\mathscr{P}(t))\left(\mathscr{I} \otimes Q_{5}\right) \widetilde{g}(\mathscr{P}(t)), \\
& \dot{\mathscr{V}}_{6}(t)=\mathscr{P}^{T}(t)\left(\mathscr{I} \otimes \mathscr{Q}_{6}\right) \mathscr{P}(t)-(1-\dot{m}(t)) \mathscr{P}^{T}(t)\left(\mathscr{I} \otimes \mathscr{Q}_{6}\right) \mathscr{P}(t), \\
& \leq \mathscr{P}^{T}(t)\left(\mathscr{I} \otimes \mathbb{Q}_{6}\right) \mathscr{P}(t)-(1-\lambda) \mathscr{P}^{T}(t)\left(\mathscr{I} \otimes \mathscr{Q}_{6}\right) \mathscr{P}(t), \\
& \dot{\mathscr{V}}_{7}(t)=\mathscr{P}^{T}(t)\left(\mathscr{I} \otimes Q_{7}\right) \mathscr{P}(t)-\mathscr{P}^{T}(t-\varrho(t))\left(\mathscr{I} \otimes Q_{7}\right) \mathscr{P}(t-\varrho(t)), \\
& \dot{\mathscr{V}}_{8}(t)=\varrho^{2} \widetilde{\mathfrak{g}}^{T}(\mathscr{P}(t))\left(\mathscr{I} \otimes Q_{8}\right) \widetilde{g}(\mathscr{P}(t))-\varrho \int_{t-\varrho(t)}^{t} \tilde{g}^{T}(\mathscr{P}(\sigma))\left(\mathscr{I} \otimes Q_{8}\right) \widetilde{g}(\mathscr{P}(\sigma)) \mathrm{d} \sigma .
\end{aligned}
$$


One can get from Lemma 2,

$$
-\varrho \int_{t-\varrho(t)}^{t} \tilde{\mathfrak{g}}^{T}(\mathscr{P}(\sigma))\left(\mathscr{I} \otimes Q_{8}\right) \tilde{g}(\mathscr{P}(\sigma)) \mathrm{d} \sigma \leq-\left(\int_{t-\varrho(t)}^{t} \tilde{\mathfrak{g}}(\mathscr{P}(\sigma)) \mathrm{d} \sigma\right)^{T}\left(\mathscr{I} \otimes Q_{8}\right)\left(\int_{t-\varrho(t)}^{t} \tilde{g}(\mathscr{P}(\sigma)) \mathrm{d} \sigma\right)
$$

In view of Assumption 1, it can be seen that $\mathscr{P}^{T}(t)\left(\mathscr{I} \otimes \mathscr{M}^{T} \mathscr{M}\right) \mathscr{P}(t)-\tilde{g}^{T}(\mathscr{P}(t)) \widetilde{g}(\mathscr{P}(t)) \geq 0$.
In addition, the following inequality applies

$$
\begin{aligned}
0= & 2[\mathscr{P}(t)-\omega \dot{\mathscr{P}}(t)]^{T}(\mathscr{I} \otimes \mathscr{H})[\dot{\mathscr{P}}(t)-\dot{\mathscr{P}}(t)] \\
= & 2[\mathscr{P}(t)-\omega \dot{\mathscr{P}}(t)]^{T}(\mathscr{I} \otimes \mathscr{H})[\dot{\mathscr{P}}(t)+(\mathscr{L} \otimes \mathscr{A}) \mathscr{P}(t)-(\mathscr{I} \otimes \mathscr{B}) \widetilde{g}(\mathscr{P}(t)) \\
& \left.-(\mathscr{I} \otimes \mathscr{C}) \widetilde{g}(\mathscr{P}(t-m(t)))-(\mathscr{I} \otimes \mathscr{D}) \int_{t-\varrho(t)}^{t} \tilde{g}(\mathscr{P}(\sigma)) \mathrm{d} \sigma\right] \\
= & 2 \mathscr{P}^{T}(t)(\mathscr{I} \otimes \mathscr{H}) \dot{\mathscr{P}}(t)-2 \mathscr{P}^{T}(t)(\mathscr{L} \otimes \mathscr{H} \mathscr{A}) \mathscr{P}(t)-2 \mathscr{P}^{T}(t)(\mathscr{I} \otimes \mathscr{H} \mathscr{B}) \tilde{g}(\mathscr{P}(t)) \\
& -2 \mathscr{P}^{T}(t)(\mathscr{I} \otimes \mathscr{H} \mathscr{C}) \tilde{g}(\mathscr{P}(t-m(t)))-2 \mathscr{P}^{T}(t)(\mathscr{I} \otimes \mathscr{H} \mathscr{D}) \int_{\varrho}^{t} \tilde{g}((\mathscr{P}(\sigma)) \mathrm{d} \sigma) \\
& +2 \omega \dot{\mathscr{P}}^{T}(t)(\mathscr{I} \otimes \mathscr{H} \mathscr{C}) \widetilde{g}(\mathscr{P}(t-m(t)))+2 \omega \dot{\mathscr{P}}^{T}(t)(\mathscr{I} \otimes \mathscr{H} \mathscr{D}) \int_{t-\varrho(t)}^{t} \widetilde{g}((\mathscr{P}(\sigma)) \mathrm{d} \sigma) \\
& -2 \omega \dot{\mathscr{P}}^{T}(t)(\mathscr{I} \otimes \mathscr{H}) \dot{\mathscr{P}}(t)+2 \omega \dot{\mathscr{P}}^{T}(t)(\mathscr{I} \otimes \mathscr{H} \mathscr{A}) \mathscr{P}(t)+2 \omega \dot{\mathscr{P}}^{T}(t)(\mathscr{I} \otimes \mathscr{H} \mathscr{B}) \widetilde{g}(\mathscr{P}(t)) .
\end{aligned}
$$

By using Lemma 1, we get

$$
\begin{aligned}
& 2 \mathscr{P}^{T}(t)\left(\mathscr{I} \otimes Q_{1} \mathscr{B}\right) \tilde{g}(\mathscr{P}(t)) \leq \omega_{1} \mathscr{P}^{T}(t)\left(\mathscr{I} \otimes Q_{1} \mathscr{B}\right)\left(\mathscr{I} \otimes Q_{1} \mathscr{B}\right)^{T} \mathscr{P}(t)+\varpi_{1}^{-1} \tilde{g}^{T}(\mathscr{P}(t)) \widetilde{g}(\mathscr{P}(t)), \\
& 2 \mathscr{P}^{T}(t)\left(\mathscr{I} \otimes \mathbb{Q}_{1} \mathscr{C}\right) \widetilde{g}(\mathscr{P}(t-m(t))) \leq \omega_{2} \mathscr{P}^{T}(t)\left(\mathscr{I} \otimes \mathbb{Q}_{1} \mathscr{C}\right)\left(\mathscr{I} \otimes \mathbb{Q}_{1} \mathscr{C}\right)^{T} \mathscr{P}(t)+\omega_{2}^{-1} \widetilde{g}^{T}(\mathscr{P}(t-m(t))) \widetilde{g}(\mathscr{P}(t-m(t)))
\end{aligned}
$$

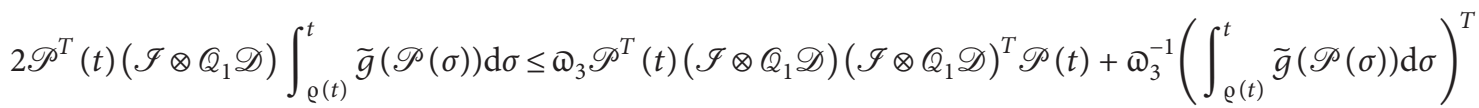

$$
\begin{aligned}
& \cdot\left(\int_{\varrho(t)}^{t} \tilde{g}(\mathscr{P}(\sigma)) \mathrm{d} \sigma\right)
\end{aligned}
$$

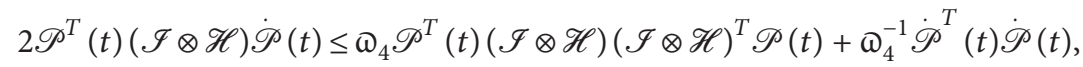

$$
\begin{aligned}
& 2 \mathscr{P}^{T}(t)(\mathscr{I} \otimes \mathscr{H} \mathscr{B}) \tilde{g}(\mathscr{P}(t)) \leq \omega_{5} \mathscr{P}^{T}(t)(\mathscr{I} \otimes \mathscr{H} \mathscr{B})(\mathscr{I} \otimes \mathscr{H} \mathscr{B})^{T} \mathscr{P}(t)+\varpi_{5}^{-1} \tilde{g}^{T}(\mathscr{P}(t)) \widetilde{g}(\mathscr{P}(t)), \\
& \cdot\left(\int_{\varrho(t)}^{t} \widetilde{g}(\mathscr{P}(\sigma)) \mathrm{d} \sigma\right)
\end{aligned}
$$

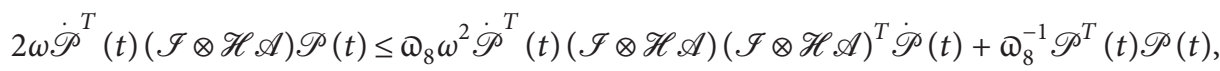




$$
\begin{aligned}
& 2 \omega \dot{\mathscr{P}}^{T}(t)(\mathscr{I} \otimes \mathscr{H} \mathscr{B}) \widetilde{g}(\mathscr{P}(t)) \leq \varpi_{9} \omega^{2} \dot{\mathscr{P}}^{T}(t)(\mathscr{I} \otimes \mathscr{H} \mathscr{B})(\mathscr{I} \otimes \mathscr{H} \mathscr{B})^{T} \mathscr{P}(t)+\varpi_{9}^{-1} \tilde{g}^{T}(\mathscr{P}(t)) \tilde{g}(\mathscr{P}(t)) \\
& 2 \omega \dot{\mathscr{P}}^{T}(t)(\mathscr{I} \otimes \mathscr{H} \mathscr{C}) \tilde{g}(\mathscr{P}(t-m(t))) \leq{\omega_{10}} \omega^{2} \dot{\mathscr{P}}^{T}(t)(\mathscr{I} \otimes \mathscr{H} \mathscr{C})(\mathscr{I} \otimes \mathscr{H} \mathscr{C})^{T} \dot{\mathscr{P}}(t)+{\varpi_{10}^{-1}}^{T}(\mathscr{P}(t-m(t))) \tilde{g}(\mathscr{P}(t-m(t))),
\end{aligned}
$$

$$
\begin{aligned}
2 \omega \dot{\mathscr{P}}^{T}(t)(\mathscr{I} \otimes \mathscr{H} \mathscr{D}) \int_{\varrho(t)}^{t} \tilde{\mathfrak{g}}(\mathscr{P}(\sigma)) \mathrm{d} \sigma & \leq \Phi_{11} \omega^{2} \dot{\mathscr{P}}^{T}(t)(\mathscr{I} \otimes \mathscr{H} \mathscr{D})(\mathscr{I} \otimes \mathscr{H} \mathscr{D})^{T} \dot{P}(t) \\
& +\oplus_{11}^{-1}\left(\int_{\varrho(t)}^{\mathrm{t}} \tilde{\mathfrak{g}}(\mathscr{P}(\sigma)) \mathrm{d} \sigma\right)^{T}\left(\int_{\varrho(t)}^{t} \tilde{\mathfrak{g}}(\mathscr{P}(\sigma)) \mathrm{d} \sigma\right) .
\end{aligned}
$$

Combining (19)-(39), we obtain

$$
\begin{aligned}
\dot{\mathscr{V}}(t) \leq & \xi^{T}(t) \Delta \xi(t) \\
\xi^{T}(t)= & {\left[\mathscr{P}^{T}(t) \mathscr{P}^{T}(t-m(t)) \mathscr{P}^{T}\left(t-m_{1}\right) \mathscr{P}^{T}\left(t-m_{2}\right) \dot{P}(t) g^{T}(\mathscr{P}(t))\right.} \\
& \left.g^{T}(\mathscr{P}(t-m(t))) \mathscr{P}^{T}(t-\varrho(t)) \int_{\varrho(t)}^{t} g^{T}(\mathscr{P}(\sigma)) \mathrm{d} \sigma\right] .
\end{aligned}
$$

As a consequence, Lyapunov stability theory analysis and based on the above argument, the equilibrium point of the multi-agent systems is asymptotically stable.

Remark 2. For consequence, in Theorem 1, the time delaydependent asymptotic stability criteria for MASs (14) can be entrenched by utilizing the novel Lyapunov functions.

Remark 3. In system (15), if there is no discrete and distributed delay occurs (i.e., $m(t)=0, \varrho(t)=0$ ), the multiagent systems will then be reduced to the next multi-agent systems.

$$
\dot{\mathscr{P}}(t)=-(\mathscr{L} \otimes \mathscr{A}) \mathscr{P}(t)+(\mathscr{I} \otimes \mathscr{B}) \widetilde{g}(\mathscr{P}(t)) .
$$

The following corollary has been designed ready for this system.

Corollary 1. The system (41) can be achieved asymptotically stable if there exists positive scalars $\Phi_{\mathfrak{\Omega}}$, where $\mathfrak{\Omega}=$ $1, \ldots, 5$ and positive definite matrices $\mathbb{Q}_{1} \in \mathscr{R}^{n \times n}$, and any matrix $\mathscr{H}$ is holding the following inequality:

$$
\begin{aligned}
- & 2\left(\mathscr{L} \otimes \mathscr{Q}_{1} \mathscr{A}\right)-2(\mathscr{I} \otimes \mathscr{H} \mathscr{A})+\varpi_{1}\left(\mathscr{I} \otimes Q_{1} \mathscr{B}\right)\left(\mathscr{I} \otimes \mathscr{Q}_{1} \mathscr{B}\right)^{T}+\left(\mathscr{I} \otimes \mathscr{M}^{T} \mathscr{M}\right)+\varpi_{2}(\mathscr{I} \otimes \mathscr{H})(\mathscr{I} \otimes \mathscr{H})^{T} \\
& -\varpi_{3}(\mathscr{I} \otimes \mathscr{H} \mathscr{B})(\mathscr{I} \otimes \mathscr{H} \mathscr{B})^{T}+\varpi_{4}^{-1}<0, \\
& \varpi_{1}^{-1}-\varpi_{3}^{-1}+\varpi_{5}^{-1}-1<0 \\
& -2 \omega(\mathscr{I} \otimes \mathscr{H})+\Phi_{2}^{-1}-\omega^{2} \varpi_{4}(\mathscr{L} \otimes \mathscr{H} \mathscr{A})(\mathscr{L} \otimes \mathscr{H} \mathscr{A})^{T}+\omega^{2} \varpi_{5}(\mathscr{I} \otimes \mathscr{H} \mathscr{B})(\mathscr{I} \otimes \mathscr{H} \mathscr{B})^{T}<0 .
\end{aligned}
$$

Remark 4. In system (15), if there is discrete delay but no distributed time-varying delay occurs (i.e., $m(t) \neq 0$, $\varrho(t)=0$ ), the multiagent system would then be transformed into the following multiagent system.

$$
\begin{aligned}
\dot{\mathscr{P}}(t)= & -(\mathscr{L} \otimes \mathscr{A}) \mathscr{P}(t)+(\mathscr{I} \otimes \mathscr{B}) \tilde{g}(\mathscr{P}(t)) \\
& +(\mathscr{I} \otimes \mathscr{C}) \tilde{g}(\mathscr{P}(t-m(t))) .
\end{aligned}
$$

The following corollary has designed readily for the above system.

Corollary 2. The system (43) can be achieved asymptotically stable for given positive constants $m_{1}, m_{2}$, and $\lambda$ if there exists positive scalars $\omega$ and $\Phi_{\mathfrak{K}}$, where $\mathfrak{K}=1, \ldots, 8$ and positive definite matrices $\mathbb{Q}_{\mathfrak{K}} \in \mathscr{R}^{n \times n}$, where $\mathfrak{K}=1, \ldots, 6$, and any matrix $\mathscr{H}$ is holding the following inequality: 


$$
\Delta=\left[\begin{array}{ccccccc}
\bar{\Omega}_{11} & 0 & 0 & 0 & 0 & 0 & 0 \\
\star & \bar{\Omega}_{22} & 0 & 0 & 0 & 0 & 0 \\
\star & \star & \bar{\Omega}_{33} & 0 & 0 & 0 & 0 \\
\star & \star & \star & \bar{\Omega}_{44} & 0 & 0 & 0 \\
\star & \star & \star & \star & \bar{\Omega}_{55} & 0 & 0 \\
\star & \star & \star & \star & \star & \bar{\Omega}_{66} & 0 \\
\star & \star & \star & \star & \star & \star & \bar{\Omega}_{77}
\end{array}\right]<0
$$

where

$$
\begin{aligned}
& \bar{\Omega}_{11}=-2\left(\mathscr{L} \otimes \mathscr{Q}_{1} \mathscr{A}\right)+m_{1}\left(\mathscr{I} \otimes \mathscr{Q}_{2}\right)+m_{2}\left(\mathscr{I} \otimes \mathscr{Q}_{3}\right)+\left(\mathscr{I} \otimes \mathscr{Q}_{6}\right)+\left(\mathscr{I} \otimes \mathscr{M}^{T} \mathscr{M}\right)+\varpi_{1}\left(\mathscr{I} \otimes Q_{1} \mathscr{B}\right)\left(\mathscr{I} \otimes \mathscr{Q}_{1} \mathscr{B}\right)^{T} \\
& +\varpi_{2}\left(\mathscr{I} \otimes Q_{1} \mathscr{C}\right)\left(\mathscr{I} \otimes \mathscr{Q}_{1} \mathscr{C}\right)^{T}+\varpi_{4}(\mathscr{I} \otimes \mathscr{H})(\mathscr{I} \otimes \mathscr{H})^{T}-\varpi_{5}(\mathscr{I} \otimes \mathscr{H} \mathscr{B})(\mathscr{I} \otimes \mathscr{H} \mathscr{B})^{T} \\
& -\varpi_{6}(\mathscr{I} \otimes \mathscr{H} \mathscr{C})(\mathscr{I} \otimes \mathscr{H} \mathscr{C})^{T}+\omega_{8}^{-1}-2(\mathscr{I} \otimes \mathscr{H} \mathscr{A}), \\
& \bar{\Omega}_{22}=-(1-\lambda)\left(\mathscr{I} \otimes Q_{6}\right) \text {, } \\
& \bar{\Omega}_{33}=-m_{1}\left(\mathscr{I} \otimes \mathbb{Q}_{2}\right)+\left(\mathscr{I} \otimes \mathbb{Q}_{4}\right), \\
& \bar{\Omega}_{44}=-m_{2}\left(\mathscr{I} \otimes \mathscr{Q}_{3}\right)-\left(\mathscr{I} \otimes \mathscr{Q}_{4}\right) \text {, } \\
& \bar{\Omega}_{55}=-2 \omega(\mathscr{I} \otimes \mathscr{H})-\varpi_{8} \omega^{2}(\mathscr{L} \otimes \mathscr{H} \mathscr{A})(\mathscr{L} \otimes \mathscr{H} \mathscr{A})^{T}+\varpi_{3} \omega^{2}(\mathscr{I} \otimes \mathscr{H} \mathscr{B})(\mathscr{I} \otimes \mathscr{H} \mathscr{B})^{T}+\omega_{4}^{-1}+\omega_{7} \omega^{2}(\mathscr{I} \otimes \mathscr{H} \mathscr{C})(\mathscr{I} \otimes \mathscr{H} \mathscr{C})^{T}, \\
& \bar{\Omega}_{66}=-1+\left(\mathscr{I} \otimes Q_{5}\right)+\varpi_{1}^{-1}-\oplus_{5}^{-1}+\varpi_{3}^{-1} \text {, } \\
& \bar{\Omega}_{77}=-(1-\lambda)\left(\mathscr{I} \otimes Q_{5}\right)+\Phi_{2}^{-1}-\varpi_{6}^{-1}+\varpi_{7}^{-1} \text {. }
\end{aligned}
$$

Remark 5. From the above section, one can ensure that the system stability is attained, if all the agents in a leaderless multiagent system should integrate the job within themselves to achieve the required criteria without designing any controllers into the system.

If the leaderless multiagent system fails to fulfill the stability criteria, then assign any one of the agents as a leader in the system and coordinate all those agents to achieve their goals. For this case, few controllers have to be designed, and this criteria can be obtained by using the synchronization analysis in the following section.

\section{Synchronization Criteria}

In this section, we will investigate the synchronization criteria for mixed time delays with leader and controller of the follower problem for the nonlinear MASs.

Now, we consider the leader system. The corresponding follower system is given by

$$
\left\{\begin{array}{l}
\dot{\mathcal{U}}_{0}(t)=\mathscr{A} \mathscr{U}_{0}(t)+\mathscr{B} \widetilde{h}\left(\mathscr{U}_{0}(t)\right)+\mathscr{C} \widetilde{h}\left(\mathscr{U}_{0}(t-m(t))\right)+\mathscr{D} \int_{t-\varrho(t)}^{t} \widetilde{h}\left(\mathscr{U}_{0}(\sigma)\right) \mathrm{d} \sigma \\
\mathcal{U}_{0}(0)=\mathscr{U}_{0}^{0}(t),
\end{array}\right.
$$

where $\mathscr{U}_{0}(t) \in \mathscr{R}^{n}$ is the state of the leader agent; $\widetilde{h}: \mathscr{R}^{n} \longrightarrow \mathscr{R}^{n}$ describes the intrinsic dynamics of each and every agents.

$$
\left\{\begin{array}{l}
\dot{U}_{i}(t)=\mathscr{A} \mathscr{U}_{i}(t)+\mathscr{B} \tilde{h}\left(\mathscr{U}_{i}(t)\right)+\mathscr{C} \tilde{h}\left(\mathcal{U}_{i}(t-m(t))\right)+\mathscr{D} \int_{t-\varrho(t)}^{t} \tilde{h}\left(\mathscr{U}_{i}(\sigma)\right) \mathrm{d} \sigma+\mathscr{K}_{i}(t), \\
\mathscr{U}_{i}(t)=\mathscr{U}_{i}^{0}(t), \quad t \in[-\widehat{\eta}, 0],
\end{array}\right.
$$


where $i=1,2,3, \ldots, N ; \mathscr{U}_{i}(t) \in \mathscr{R}^{n}$ is the $i^{\text {th }}$ agent state; $\widetilde{h}: \mathscr{R}^{n} \longrightarrow \mathscr{R}^{n}$ describes the intrinsic dynamics of each agent. Let $\mathscr{A}, \mathscr{B}, \mathscr{C}$, and $\mathscr{D}$ are the matrices of $\mathscr{R}^{n \times n} \cdot m(t)$ is the discrete delay, and $\varrho(t)$ is the distributed delay that satisfies $0 \leq m_{1} \leq m(t) \leq m_{2}, \dot{m}(t) \leq \lambda, 0<\varrho(t) \leq \varrho$, where $m_{1}, m_{2}, \lambda$, and $\varrho$ are the constants, and $\hat{\eta}=\max \left\{m_{1}, m_{2}, \varrho\right\}$. Let $\delta_{i}(t)=\mathscr{U}_{i}(t)-\mathscr{U}_{0}(t)$.

Then, $\dot{\mathcal{S}}_{i}(t)=\dot{\mathscr{U}}_{i}(t)-\dot{\mathscr{U}}_{0}(t)$. The synchronization error system is given by

$$
\left\{\begin{array}{l}
\dot{\mathcal{S}}_{i}(t)=\mathscr{A} \mathcal{S}_{i}(t)+\mathscr{B} \tilde{g}\left(\mathcal{S}_{i}(t)\right)+\mathscr{C} \tilde{g}\left(\mathcal{S}_{i}(t-m(t))\right)+\mathscr{D} \int_{t-\varrho(t)}^{t} \tilde{g}\left(\mathcal{S}_{i}(\sigma)\right) \mathrm{d} \sigma+\mathscr{K}_{i}(t), \\
\mathcal{S}_{i}(t)=\mathscr{U}_{i}^{0}(t)-\mathscr{U}_{0}^{0}(t), \quad \mathrm{t} \in[-\widehat{\eta}, 0],
\end{array}\right.
$$

where $i=1,2,3, \ldots, N ; \mathcal{S}_{i}(t) \in \mathscr{R}^{n}$ is the $i^{\text {th }}$ agent state; $\tilde{g}: \mathscr{R}^{n} \longrightarrow \mathscr{R}^{n}$ describes the intrinsic dynamics of each agent; $\mathscr{K}_{i}(t) \in \mathscr{R}^{m}$ is the controller. Let $\mathscr{A}, \mathscr{B}, \mathscr{C}$, and $\mathscr{D}$ are the constant matrices of $\mathscr{R}^{n \times n} . m(t)$ is the discrete timevarying delay, and $\varrho(t)$ is the distributed delay that satisfies $0 \leq m_{1} \leq m(t) \leq m_{2}, \dot{m}(t) \leq \lambda$, and $0<\varrho(t) \leq \varrho$, where $m_{1}, m_{2}$, $\lambda$, and $\varrho$ are the constants, and $\hat{\eta}=\max \left\{m_{1}, m_{2}, \varrho\right\}$, where $\tilde{g}(\mathcal{S}(t))=\widetilde{h}\left(\mathcal{U}_{i}(t)\right)-\widetilde{h}\left(\mathcal{U}_{0}(t)\right)$. Now, the controller can be designed to be of the following form:

$$
\begin{aligned}
& \mathscr{K}_{i}(t)=\mathscr{J} \sum_{k=1}^{N} \mathfrak{a}_{i k}\left(\mathcal{U}_{k}(t)-\mathscr{U}_{i}(t)\right), \\
& \mathscr{K}_{i}(t)=-\mathcal{J} \sum_{k=1}^{N} \mathfrak{l}_{i k}\left(\mathcal{S}_{k}(t)\right) .
\end{aligned}
$$

Or equivalently,

$$
\mathscr{K}(t)=-(\mathscr{L} \otimes \mathscr{J})(\mathcal{S}(t)) .
$$

where $\mathscr{J}$ is the control gain matrix.

The compact variant of the error system (50) is given as

$$
\begin{aligned}
\dot{\mathcal{S}}(t)= & (\mathscr{I} \otimes \mathscr{A}) \mathcal{S}(t)+(\mathscr{I} \otimes \mathscr{B}) \widetilde{g}(\mathcal{S}(t))+(\mathscr{I} \otimes \mathscr{C}) \tilde{g}(\mathcal{S}(t-m(t))) \\
& +(\mathscr{I} \otimes \mathscr{D}) \int_{t-\varrho(t)}^{t} \tilde{g}(\mathcal{S}(\sigma)) \mathrm{d} \sigma-(\mathscr{L} \otimes \mathscr{J}) \mathcal{S}(t),
\end{aligned}
$$

where $\mathcal{S}(t)=\left[\mathcal{S}_{1}, \mathcal{S}_{2}, \ldots, \mathcal{S}_{N}\right]^{T}$ denotes the error vector.

Theorem 2. The leader-follower system (51) can be achieved asymptotically synchronized for given positive constants $m_{1}, m_{2}$, $\varrho$, and $\lambda$ if there exists positive scalars $\omega$ and $\Phi_{\Re}$, where $\Re=1$, $\ldots, 12$ and positive matrices $\mathbb{Q}_{\mathfrak{K}} \in \mathscr{R}^{n \times n}$, where $\mathfrak{K}=1, \ldots, 8$, and any matrix $\mathscr{H}$ is holding the following inequality:

$$
\Xi=\left[\begin{array}{ccccccccc}
\bar{\Omega}_{11} & 0 & 0 & 0 & 0 & 0 & 0 & 0 & 0 \\
\star & \bar{\Omega}_{22} & 0 & 0 & 0 & 0 & 0 & 0 & 0 \\
\star & \star & \bar{\Omega}_{33} & 0 & 0 & 0 & 0 & 0 & 0 \\
\star & \star & \star & \bar{\Omega}_{44} & 0 & 0 & 0 & 0 & 0 \\
\star & \star & \star & \star & \bar{\Omega}_{55} & 0 & 0 & 0 & 0 \\
\star & \star & \star & \star & \star & \bar{\Omega}_{66} & 0 & 0 & 0 \\
\star & \star & \star & \star & \star & \star & \bar{\Omega}_{77} & 0 & 0 \\
\star & \star & \star & \star & \star & \star & \star & \bar{\Omega}_{88} & 0 \\
\star & \star & \star & \star & \star & \star & \star & \star & \bar{\Omega}_{99}
\end{array}\right]<0,
$$


where

$$
\begin{aligned}
& \bar{\Omega}_{11}=2\left(\mathscr{I} \otimes \mathscr{Q}_{1} \mathscr{A}\right)+m_{1}\left(\mathscr{I} \otimes \mathscr{Q}_{2}\right)+m_{2}\left(\mathscr{I} \otimes \mathbb{Q}_{3}\right)+\left(\mathscr{I} \otimes \mathbb{Q}_{6}\right)+\left(\mathscr{I} \otimes \mathbb{Q}_{7}\right)+\left(\mathscr{I} \otimes \mathscr{M}^{T} \mathscr{M}\right) \\
& +\varpi_{1}\left(\mathscr{I} \otimes \mathscr{Q}_{1} \mathscr{B}\right)\left(\mathscr{I} \otimes \mathscr{Q}_{1} \mathscr{B}\right)^{T}+\varpi_{2}\left(\mathscr{I} \otimes Q_{1} \mathscr{C}\right)\left(\mathscr{I} \otimes Q_{1} \mathscr{C}\right)^{T}+\varpi_{3}\left(\mathscr{I} \otimes Q_{1} \mathscr{D}\right)\left(\mathscr{I} \otimes Q_{1} \mathscr{D}\right)^{T} \\
& +\omega_{4}(\mathscr{I} \otimes \mathscr{H})(\mathscr{I} \otimes \mathscr{H})^{T}-\omega_{5}(\mathscr{I} \otimes \mathscr{H} \mathscr{B})(\mathscr{I} \otimes \mathscr{H} \mathscr{B})^{T}-\omega_{6}(\mathscr{I} \otimes \mathscr{H} \mathscr{C})(\mathscr{I} \otimes \mathscr{H} \mathscr{C})^{T} \\
& -\omega_{7}(\mathscr{I} \otimes \mathscr{H} \mathscr{D})(\mathscr{I} \otimes \mathscr{H} \mathscr{D})^{T}+\varpi_{8}^{-1}-2(\mathscr{I} \otimes \mathscr{H} \mathscr{A})+2\left(\mathscr{L} \otimes \mathscr{Q}_{1} \mathscr{J}\right)-2(\mathscr{L} \otimes \mathscr{H} \mathscr{J})-\varpi_{12}^{-1}, \\
& \bar{\Omega}_{22}=-(1-\lambda)\left(\mathscr{I} \otimes Q_{6}\right) \text {, } \\
& \bar{\Omega}_{33}=-m_{1}\left(\mathscr{I} \otimes \mathbb{Q}_{2}\right)+\left(\mathscr{I} \otimes \mathbb{Q}_{4}\right) \text {, } \\
& \bar{\Omega}_{44}=-m_{2}\left(\mathscr{I} \otimes \mathscr{Q}_{3}\right)-\left(\mathscr{I} \otimes \mathscr{Q}_{4}\right) \text {, } \\
& \bar{\Omega}_{55}=-2 \omega(\mathscr{I} \otimes \mathscr{H})+\omega_{8} \omega^{2}(\mathscr{I} \otimes \mathscr{H} \mathscr{A})(\mathscr{I} \otimes \mathscr{H} \mathscr{A})^{T}+\omega_{9} \omega^{2}(\mathscr{I} \otimes \mathscr{H} \mathscr{B})(\mathscr{I} \otimes \mathscr{H} \mathscr{B})^{T}+\omega_{4}^{-1} \\
& +\varpi_{10} \omega^{2}(\mathscr{I} \otimes \mathscr{H} \mathscr{C})(\mathscr{I} \otimes \mathscr{H} \mathscr{C})^{T}+\omega_{11} \omega^{2}(\mathscr{I} \otimes \mathscr{H} \mathscr{D})(\mathscr{I} \otimes \mathscr{H} \mathscr{D})^{T}-\varpi_{12} \omega^{2}(\mathscr{L} \otimes \mathscr{H} \mathscr{J})(\mathscr{L} \otimes \mathscr{H} \mathscr{J})^{T}, \\
& \bar{\Omega}_{66}=-1+\left(\mathscr{I} \otimes Q_{5}\right)+\varrho^{2}\left(\mathscr{I} \otimes Q_{8}\right)+\varpi_{1}^{-1}-\omega_{5}^{-1}+\varpi_{9}^{-1}, \\
& \bar{\Omega}_{77}=-(1-\lambda)\left(\mathscr{I} \otimes Q_{5}\right)+\omega_{2}^{-1}-\omega_{6}^{-1}+\omega_{10}^{-1} \text {, } \\
& \bar{\Omega}_{88}=-\left(\mathscr{I} \otimes Q_{7}\right) \text {, } \\
& \bar{\Omega}_{99}=-\left(\mathscr{I} \otimes Q_{8}\right)+\varpi_{3}^{-1}+\varpi_{11}^{-1}-\varpi_{7}^{-1} .
\end{aligned}
$$

Proof. Construct the following function of Lyapunov-Krasovskii candidate:

$$
\mathscr{V}(t)=\sum_{i=1}^{8} \mathscr{V}(t)_{i}
$$

where $\mathscr{V}_{1}(t)=\mathcal{S}^{T}(t)\left(\mathscr{I} \otimes Q_{1}\right) \mathcal{S}(t)$,

$$
\begin{aligned}
& \mathscr{V}_{2}(t)=m_{1} \int_{t-m_{1}}^{t} \mathcal{S}^{T}(\sigma)\left(\mathscr{I} \otimes \mathbb{Q}_{2}\right) \mathcal{S}(\sigma) \mathrm{d} \sigma, \\
& \mathscr{V}_{3}(t)=m_{2} \int_{t-m_{2}}^{t} \mathscr{P}^{T}(\sigma)\left(\mathscr{I} \otimes \mathscr{Q}_{3}\right) \mathcal{S}(\sigma) \mathrm{d} \sigma \\
& \mathscr{V}_{4}(t)=\int_{t-m_{2}}^{t-m_{1}} \mathcal{S}^{T}(\sigma)\left(\mathscr{I} \otimes Q_{4}\right) \mathcal{S}(\sigma) \mathrm{d} \sigma, \\
& \mathscr{V}_{5}(t)=\int_{t-m(t)}^{t} \tilde{g}^{T}(\mathcal{S}(\sigma))\left(\mathscr{I} \otimes Q_{5}\right) \tilde{g}(\mathcal{S}(\sigma)) \mathrm{d} \sigma, \\
& \mathscr{V}_{6}(t)=\int_{t-m(t)}^{t} \mathcal{S}^{T}(\sigma)\left(\mathscr{I} \otimes \mathscr{Q}_{6}\right) \mathcal{S}(\sigma) \mathrm{d} \sigma, \\
& \mathscr{V}_{7}(t)=\int_{t-\varrho(t)}^{t} \mathcal{S}^{T}(\sigma)\left(\mathscr{I} \otimes Q_{7}\right) \mathcal{S}(\sigma) \mathrm{d} \sigma, \\
& \mathscr{V}_{8}(t)=\varrho \int_{-\varrho(t)}^{0} \int_{t+\theta}^{t} \tilde{g}^{T}(\mathcal{S}(\sigma))\left(\mathscr{I} \otimes \mathbb{Q}_{8}\right) \tilde{g}(\mathcal{S}(\sigma)) \mathrm{d} \sigma \mathrm{d} \theta .
\end{aligned}
$$


The time derivative of (54) will then be

$$
\begin{aligned}
& \dot{\mathscr{V}}_{1}(t)=2 \mathcal{S}^{T}(t)\left(\mathscr{I} \otimes Q_{1}\right) \dot{\mathcal{S}}(t) \\
& \dot{\mathscr{V}}_{1}(t)=2 \mathcal{S}^{T}(t)\left(\mathscr{I} \otimes \mathbb{Q}_{1}\right)[(\mathscr{I} \otimes \mathscr{A}) \mathcal{S}(t)+(\mathscr{I} \otimes \mathscr{B}) \widetilde{g}(\mathcal{S}(t)) \\
& \left.+(\mathscr{I} \otimes \mathscr{C}) \widetilde{g}(\mathcal{S}(t-m(t)))+(\mathscr{I} \otimes \mathscr{D}) \int_{\mathrm{t}-\varrho(t)}^{t} \tilde{g}(\mathcal{S}(\sigma)) \mathrm{d} \sigma-(\mathscr{L} \otimes \mathscr{J}) \mathcal{S}(t)\right] \\
& =2 \mathcal{S}^{T}(t)\left(\mathscr{I} \otimes \mathbb{Q}_{1} \mathscr{A}\right) \mathcal{S}(t)+2 \mathcal{S}^{T}(t)\left(\mathscr{I} \otimes Q_{1} \mathscr{B}\right) \widetilde{g}(\mathcal{S}(t))+2 \mathcal{S}^{T}(t)\left(\mathscr{I} \otimes \mathscr{Q}_{1} \mathscr{C}\right) \tilde{g}(\mathcal{S}(t-m(t))) \\
& +2 \mathcal{S}^{T}(t)\left(\mathscr{I} \otimes Q_{1} \mathscr{D}\right) \int_{\mathrm{t}-\varrho(t)}^{t} \tilde{g}(\mathcal{S}(\sigma)) \mathrm{d} \sigma+2 \mathcal{S}^{T}(t)\left(\mathscr{L} \otimes Q_{1} \mathscr{J}\right) \mathcal{S}(t) \\
& \dot{\mathscr{V}}_{2}(t)=m_{1} \mathcal{S}^{T}(t)\left(\mathscr{I} \otimes Q_{2}\right) \mathcal{S}(t)-m_{1} \mathscr{P}^{T}\left(t-m_{1}\right)\left(\mathscr{I} \otimes Q_{2}\right) \mathcal{S}\left(t-m_{1}\right) \\
& \dot{\mathscr{V}}_{3}(t)=m_{2} \mathcal{S}^{T}(t)\left(\mathscr{I} \otimes Q_{3}\right) \mathcal{S}(t)-m_{2} \mathcal{S}^{T}\left(t-m_{2}\right)\left(\mathscr{I} \otimes Q_{3}\right) \mathcal{S}\left(t-m_{2}\right), \\
& \dot{\mathscr{V}}_{4}(t)=\mathcal{S}^{T}\left(t-m_{1}\right)\left(\mathscr{I} \otimes Q_{4}\right) \mathcal{S}\left(t-m_{1}\right)-\mathcal{S}^{T}\left(t-m_{2}\right)\left(\mathscr{I} \otimes Q_{4}\right) \mathcal{S}\left(t-m_{2}\right) \\
& \dot{\mathscr{V}}_{5}(t)=\tilde{g}^{T}(\mathcal{S}(t))\left(\mathscr{I} \otimes \widehat{Q}_{5}\right) \widetilde{g}(\mathcal{S}(t))-(1-\dot{m}(t)) \tilde{g}^{T}(\mathcal{S}(t))\left(\mathscr{I} \otimes \mathscr{Q}_{5}\right) \widetilde{g}(\mathcal{S}(t)) \\
& \leq \widetilde{g}^{T}(\mathcal{S}(t))\left(\mathscr{I} \otimes Q_{5}\right) \tilde{g}(\mathcal{S}(t))-(1-\lambda) \tilde{g}^{T}(\mathcal{S}(t))\left(\mathscr{I} \otimes Q_{5}\right) \widetilde{g}(\mathcal{S}(t)), \\
& \dot{\mathscr{V}}_{6}(t)=\mathcal{S}^{T}(t)\left(\mathscr{I} \otimes Q_{6}\right) \mathcal{S}(t)-(1-\dot{m}(t)) \mathcal{S}^{T}(t)\left(\mathscr{I} \otimes Q_{6}\right) \mathcal{S}(t) \\
& \leq \mathcal{S}^{T}(t)\left(\mathscr{I} \otimes Q_{6}\right) \mathcal{S}(t)-(1-\lambda) \mathcal{S}^{T}(t)\left(\mathscr{I} \otimes Q_{6}\right) \mathcal{S}(t), \\
& \dot{\mathscr{V}}_{7}(t)=\mathcal{S}^{T}(t)\left(\mathscr{I} \otimes Q_{7}\right) \mathcal{S}(t)-\mathcal{S}^{T}(t-\varrho(t))\left(\mathscr{I} \otimes Q_{7}\right) \mathcal{S}(t-\varrho(t)), \\
& \dot{\mathscr{V}}_{8}(t)=\varrho^{2} \tilde{g}^{T}(\mathcal{S}(t))\left(\mathscr{I} \otimes Q_{8}\right) \tilde{g}(\mathcal{S}(t))-\varrho \int_{t-\varrho(t)}^{t} \tilde{g}^{T}(\mathcal{S}(\sigma))\left(\mathscr{I} \otimes \mathscr{Q}_{8}\right) \tilde{g}(\mathcal{S}(\sigma)) \mathrm{d} \sigma .
\end{aligned}
$$

By using Lemma 2, we have

$$
\begin{aligned}
& -\varrho \int_{t-\varrho(t)}^{t} \widetilde{\mathfrak{g}}^{T}(\mathcal{S}(\sigma))\left(\mathscr{I} \otimes Q_{8}\right) \widetilde{g}(\mathcal{S}(\sigma)) \mathrm{d} \sigma \\
& \quad \leq-\left(\int_{t-\varrho(t)}^{t} \tilde{\mathfrak{g}}(\mathcal{S}(\sigma)) \mathrm{d} \sigma\right)^{T}\left(\mathscr{I} \otimes \mathscr{Q}_{8}\right)\left(\int_{\mathrm{t}-\varrho(t)}^{t} \widetilde{g}(\mathcal{S}(\sigma)) \mathrm{d} \sigma\right) .
\end{aligned}
$$

In view of Assumption 1, it can be seen that $\mathcal{S}^{T}(t)\left(\mathscr{I} \otimes \mathscr{M}^{T} \mathscr{M}\right) \mathcal{S}(t)-\widetilde{g}^{T}(\mathcal{S}(t)) \widetilde{g}(\mathcal{S}(t)) \geq 0$.

$$
\begin{aligned}
& 0=2[\mathcal{S}(t)-\omega \dot{\mathcal{S}}(t)]^{T}(\mathscr{I} \otimes \mathscr{H})[\dot{\mathcal{S}}(t)-\dot{\mathcal{S}}(t)] \\
& =2[\mathcal{S}(t)-\omega \dot{\mathcal{S}}(t)]^{T}(\mathscr{I} \otimes \mathscr{H})[\dot{\mathcal{S}}(t)-(\mathscr{I} \otimes \mathscr{A}) \mathcal{S}(t)-(\mathscr{I} \otimes \mathscr{B}) \widetilde{g}(\mathcal{S}(t)) \\
& \left.-(\mathscr{I} \otimes \mathscr{C}) \tilde{g}(\mathcal{S}(t-m(t)))-(\mathscr{I} \otimes \mathscr{D}) \int_{t-\varrho(t)}^{t} \tilde{g}(\mathcal{S}(\sigma)) \mathrm{d} \sigma+(\mathscr{L} \otimes \mathscr{J}) \mathcal{S}(t)\right] \\
& =2 \mathcal{S}^{T}(t)(\mathscr{I} \otimes \mathscr{H}) \dot{\mathcal{S}}(t)-2 \mathcal{S}^{T}(t)(\mathscr{I} \otimes \mathscr{H} \mathscr{A}) \mathcal{S}(t)-2 \mathcal{S}^{T}(t)(\mathscr{I} \otimes \mathscr{H} \mathscr{B}) \widetilde{g}(\mathcal{S}(t)) \\
& -2 \mathcal{S}^{T}(t)(\mathscr{I} \otimes \mathscr{H} \mathscr{C}) \tilde{g}(\mathcal{S}(t-m(t)))-2 \mathcal{S}^{T}(t)(\mathscr{I} \otimes \mathscr{H} \mathscr{D}) \int_{\varrho(t)}^{\mathrm{t}} \tilde{\mathcal{g}}(\mathcal{S}(\sigma)) \mathrm{d} \sigma-2 \mathcal{S}^{T}(t)(\mathscr{L} \otimes \mathscr{H} \mathscr{J}) \mathcal{S}(t) \\
& -2 \omega \dot{\mathcal{S}}^{T}(t)(\mathscr{I} \otimes \mathscr{H}) \dot{\mathcal{S}}(t)+2 \omega \dot{\mathcal{S}}^{T}(t)(\mathscr{I} \otimes \mathscr{H} \mathscr{A}) \mathcal{S}(t)+2 \omega \dot{\mathcal{S}}^{T}(t)(\mathscr{I} \otimes \mathscr{H} \mathscr{B}) \tilde{g}(\mathcal{S}(t)) \\
& +2 \omega \dot{\mathcal{S}}^{T}(t)(\mathscr{I} \otimes \mathscr{H} \mathscr{C}) \tilde{g}(\mathcal{S}(t-m(t)))+2 \omega \dot{\mathcal{S}}^{T}(t)(\mathscr{I} \otimes \mathscr{H} \mathscr{D}) \int_{\mathrm{t}-\varrho(t)}^{t} \tilde{\mathcal{g}}(\mathcal{S}(\sigma)) \mathrm{d} \sigma-2 \omega \dot{\mathcal{S}}^{T}(t)(\mathscr{L} \otimes \mathscr{H} \mathscr{J})(\mathcal{S}(t)) .
\end{aligned}
$$


In view of Lemma 1 , we can get

$$
\begin{aligned}
& 2 \mathcal{S}^{T}(t)\left(\mathscr{I} \otimes Q_{1} \mathscr{B}\right) \tilde{g}(\mathcal{S}(t)) \leq \omega_{1} \mathcal{S}^{T}(t)\left(\mathscr{I} \otimes Q_{1} \mathscr{B}\right)\left(\mathscr{I} \otimes Q_{1} \mathscr{B}\right)^{T} \mathcal{S}(t)+\Phi_{1}^{-1} \tilde{g}^{T}(\mathcal{S}(t)) \tilde{g}(\mathcal{S}(t)), \\
& 2 \mathcal{S}^{T}(t)\left(\mathscr{I} \otimes Q_{1} \mathscr{C}\right) \tilde{g}(\mathcal{S}(t-m(t))) \leq \omega_{2} \mathcal{S}^{T}(t)\left(\mathscr{I} \otimes Q_{1} \mathscr{C}\right)\left(\mathscr{I} \otimes Q_{1} \mathscr{C}\right)^{T} \mathcal{S}(t)+\omega_{2}^{-1} \tilde{g}^{T}(\mathcal{S}(t-m(t))) \widetilde{g}(\mathcal{S}(t-m(t))) \\
& 2 \mathcal{S}^{T}(t)\left(\mathscr{I} \otimes \mathscr{Q}_{1} \mathscr{D}\right) \int_{\varrho(t)}^{t} \tilde{\mathcal{g}}(\mathcal{S}(\sigma)) \mathrm{d} \sigma \leq \Phi_{3} \mathcal{S}^{T}(t)\left(\mathscr{I} \otimes Q_{1} \mathscr{D}\right)\left(\mathscr{I} \otimes \mathscr{Q}_{1} \mathscr{D}\right)^{T} \mathcal{S}(t)+\omega_{3}^{-1}\left(\int_{\varrho(t)}^{t} \tilde{\mathcal{g}}(\mathcal{S}(\sigma)) \mathrm{d} \sigma\right)^{T}\left(\int_{\varrho(t)}^{t} \tilde{\mathcal{g}}(\mathcal{S}(\sigma)) \mathrm{d} \sigma\right) \\
& 2 \mathcal{S}^{T}(t)(\mathscr{I} \otimes \mathscr{H}) \dot{\mathcal{S}}(t) \leq \omega_{4} \mathcal{S}^{T}(t)(\mathscr{I} \otimes \mathscr{H})(\mathscr{I} \otimes \mathscr{H})^{T} \mathcal{S}(t)+\omega_{4}^{-1} \dot{\mathcal{S}}^{T}(t) \dot{\mathcal{S}}(t), \\
& 2 \mathcal{S}^{T}(t)(\mathscr{I} \otimes \mathscr{H} \mathscr{B}) \tilde{g}(\mathcal{S}(t)) \leq \omega_{5} \mathcal{S}^{T}(t)(\mathscr{I} \otimes \mathscr{H} \mathscr{B})(\mathscr{I} \otimes \mathscr{H} \mathscr{B})^{T} \mathcal{S}(t)+\omega_{5}^{-1} \tilde{g}^{T}(\mathcal{S}(t)) \tilde{g}(\mathcal{S}(t)), \\
& 2 \mathcal{S}^{T}(t)(\mathscr{I} \otimes \mathscr{H} \mathscr{C}) \tilde{g}(\mathcal{S}(t-m(t))) \leq \omega_{6} \mathcal{S}^{T}(t)(\mathscr{I} \otimes \mathscr{H} \mathscr{C})(\mathscr{I} \otimes \mathscr{H} \mathscr{C})^{T} \mathcal{S}(t)+\varpi_{6}^{-1} \widetilde{g}^{T}(\mathcal{S}(t-m(t))) \tilde{g}(\mathcal{S}(t-m(t))) \\
& 2 \mathcal{S}^{T}(t)(\mathscr{I} \otimes \mathscr{H} \mathscr{D}) \int_{\varrho(t)}^{t} \tilde{\mathcal{g}}(\mathcal{S}(\sigma)) \mathrm{d} \sigma \leq \omega_{7} \mathcal{S}^{T}(t)(\mathscr{I} \otimes \mathscr{H} \mathscr{D})(\mathscr{I} \otimes \mathscr{H} \mathscr{D})^{T} \mathcal{S}(t)+\varpi_{7}^{-1}\left(\int_{\varrho(t)}^{t} \tilde{\mathcal{g}}(\mathcal{S}(\sigma)) \mathrm{d} \sigma\right)^{T}\left(\int_{\varrho(\mathrm{t})}^{\mathrm{t}} \tilde{\mathcal{g}}(\mathcal{S}(\sigma)) \mathrm{d} \sigma\right)
\end{aligned}
$$$$
2 \omega \dot{\mathcal{S}}^{T}(t)(\mathscr{I} \otimes \mathscr{H} \mathscr{A}) \mathcal{S}(t) \leq \omega_{8} \omega^{2} \dot{\mathcal{S}}^{T}(t)(\mathscr{I} \otimes \mathscr{H} \mathscr{A})(\mathscr{I} \otimes \mathscr{H} \mathscr{A})^{T} \dot{\mathcal{S}}(t)+\omega_{8}^{-1} \mathcal{S}^{T}(t) \mathcal{S}(t)
$$$$
2 \omega \dot{\mathcal{S}}^{T}(t)(\mathscr{I} \otimes \mathscr{H} \mathscr{B}) \widetilde{g}(\mathcal{S}(t)) \leq{\omega_{9}}^{2} \dot{\mathcal{S}}^{T}(t)(\mathscr{I} \otimes \mathscr{H} \mathscr{B})(\mathscr{I} \otimes \mathscr{H} \mathscr{B})^{T} \dot{\mathcal{S}}(t)+{\omega_{9}^{-1}}^{T}(\mathcal{g}(t)) \widetilde{g}(\mathcal{S}(t)),
$$

$2 \omega \dot{\mathcal{S}}^{T}(t)(\mathscr{I} \otimes \mathscr{H} \mathscr{C}) \widetilde{g}(\mathcal{S}(t-m(t))) \leq \omega_{10} \omega^{2} \dot{\mathscr{P}}^{T}(t)(\mathscr{I} \otimes \mathscr{H} \mathscr{C})(\mathscr{I} \otimes \mathscr{H} \mathscr{C})^{T} \dot{\mathscr{P}}(t)+\Phi_{10}^{-1} \tilde{\mathcal{g}}^{T}(\mathscr{P}(t-m(t))) \widetilde{g}(\mathscr{P}(t-m(t)))$

$2 \omega \dot{\mathcal{S}}^{T}(t)(\mathscr{I} \otimes \mathscr{H} \mathscr{D}) \int_{\varrho(t)}^{t} \widetilde{\mathcal{g}}(\mathcal{S}(\sigma)) \mathrm{d} \sigma \leq \omega_{11} \omega^{2} \dot{\mathcal{S}}^{T}(t)(\mathscr{I} \otimes \mathscr{H} \mathscr{D})(\mathscr{I} \otimes \mathscr{H} \mathscr{D})^{T} \dot{\mathcal{S}}(t)$

$$
+\omega_{11}^{-1}\left(\int_{\varrho(t)}^{t} \tilde{g}(\mathcal{S}(\sigma)) \mathrm{d} \sigma\right)^{T}\left(\int_{\varrho(t)}^{t} \tilde{g}(\mathcal{S}(\sigma)) \mathrm{d} \sigma\right)
$$

$$
2 \omega \dot{\mathcal{S}}^{T}(t)(\mathscr{L} \otimes \mathscr{H} \mathscr{J}) \mathcal{S}(t) \leq \omega_{12} \omega^{2} \dot{\mathcal{S}}^{T}(t)(\mathscr{L} \otimes \mathscr{H} \mathscr{J})(\mathscr{L} \otimes \mathscr{H} \mathscr{J})^{T} \dot{\mathcal{S}}(t)+\omega_{12}^{-1} \mathcal{S}^{T}(t) \mathcal{S}(t)
$$

Combined with (55)-(77), we obtain

$$
\begin{aligned}
\dot{\mathscr{V}}(t) \leq & \xi^{T}(t) \Xi \xi(t) \\
\text { where, } \quad \xi^{T}(t)= & {\left[\mathcal{S}^{T}(t) \mathcal{S}^{T}(t-m(t)) \mathcal{S}^{T}\left(t-m_{1}\right) \mathcal{S}^{T}\left(t-m_{2}\right) \dot{\mathcal{S}}^{T}(t) g^{T}(\mathcal{S}(t))\right.} \\
& \left.g^{T}(\mathcal{S}(t-m(t))) \mathcal{S}^{T}(t-\varrho(t)) \int_{\varrho(t)}^{t} g^{T}(\mathcal{S}(\sigma)) \mathrm{d} \sigma\right] .
\end{aligned}
$$

As a consequence, Lyapunov stability theory analysis and based on the above argument, the leader and follower multiagent systems is asymptotical synchronization.
Remark 6. In system (51), if there is no discrete and distributed delay occurs (i.e., $m(t)=0, \varrho(t)=0$ ), the multi-agent systems will then be reduced to the following multi-agent systems. 


$$
\dot{\mathcal{S}}(t)=(\mathscr{I} \otimes \mathscr{A}) \mathcal{S}(t)+(\mathscr{I} \otimes \mathscr{B}) \tilde{g}(\mathcal{S}(t))-(\mathscr{L} \otimes \mathscr{J}) \mathcal{S}(t),
$$

Then, the following corollary is readily obtained for the abovementioned system.
Corollary 3. The leader-follower system (79) can be achieved asymptotically synchronized if there exists positive scalars $\omega$ and $\Phi_{\mathfrak{K}}$, where $\Re=1,2, \ldots, 6$ and positive matrices $Q_{1} \in \mathscr{R}^{n \times n}$, and any matrix $\mathscr{H}$ is holding the following inequality:

$$
\begin{aligned}
& 2\left(\mathscr{I} \otimes Q_{1} \mathscr{A}\right)-2\left(\mathscr{L} \otimes \mathscr{Q}_{1} \mathscr{J}\right)-2(\mathscr{L} \otimes \mathscr{H} \mathscr{J})-2(\mathscr{I} \otimes \mathscr{H} \mathscr{A})+\left(\mathscr{I} \otimes \mathscr{M}^{T} \mathscr{M}\right)+\omega_{1}\left(\mathscr{I} \otimes Q_{1} \mathscr{B}\right)\left(\mathscr{I} \otimes Q_{1} \mathscr{B}\right)^{T} \\
& +\omega_{2}(\mathscr{I} \otimes \mathscr{H})(\mathscr{I} \otimes \mathscr{H})^{T}-\omega_{3}(\mathscr{I} \otimes \mathscr{H} \mathscr{B})(\mathscr{I} \otimes \mathscr{H} \mathscr{B})^{T}+\omega_{4}^{-1}-\omega_{6}^{-1}<0, \\
& \omega_{1}^{-1}-\varpi_{3}^{-1}+\varpi_{5}^{-1}-1<0 \text {, } \\
& -2 \omega(\mathscr{I} \otimes \mathscr{H})+\omega^{2} \omega_{4}(\mathscr{I} \otimes \mathscr{H} \mathscr{A})(\mathscr{I} \otimes \mathscr{H} \mathscr{A})^{T}+\omega^{2} \omega_{5}(\mathscr{I} \otimes \mathscr{H} \mathscr{B})(\mathscr{I} \otimes \mathscr{H} \mathscr{B})^{T} \\
& +\varpi_{2}^{-1}-\omega^{2} \varpi_{6}(\mathscr{L} \otimes \mathscr{H} \mathscr{J})(\mathscr{L} \otimes \mathscr{H} \mathscr{J})^{T}<0 .
\end{aligned}
$$

Remark 7. It is that the first time that MASs stability and synchronization criterion have been investigated. In this analysis, multiagent systems with nonlinear discrete timevarying delays and distributed delays are taken into account, and the results are very complicated and not easy to measure. The main achievement of this work is the generalization and resolution of this problem. Therefore, our proposed model is wider and more advanced.

Remark 8. In system (39), if there is discrete but no distributed delay occurs (i.e., $m(t) \neq 0, \varrho(t)=0$ ), the multiagent system will then be reduced as

$$
\begin{aligned}
\dot{\mathcal{S}}(t)= & (\mathscr{I} \otimes \mathscr{A}) \mathcal{S}(t)+(\mathscr{I} \otimes \mathscr{B}) \tilde{g}(\mathcal{S}(t)) \\
& +(\mathscr{I} \otimes \mathscr{C}) \tilde{g}(\mathcal{S}(t-m(t)))-(\mathscr{L} \otimes \mathscr{J}) \mathcal{S}(t) .
\end{aligned}
$$

The following corollary has been designed in order to verify the stability and synchronization for the abovementioned system.

Corollary 4. The leader-follower system (81) can be achieved asymptotically synchronized for given positive constants $m_{1}, m_{2}$, and $\lambda$ if there exists positive scalars $\omega$ and $\omega_{\Re}$, where $\mathfrak{\Omega}=1,2, \ldots, 9$ and positive definite matrices $\mathbb{Q}_{\mathfrak{K}} \in \mathscr{R}^{n \times n}$, where $\mathfrak{\Omega}=1, \ldots, 6$, and any matrix $\mathscr{H}$ is holding the following inequality:

$$
\Delta=\left[\begin{array}{ccccccc}
\bar{\Omega}_{11} & 0 & 0 & 0 & 0 & 0 & 0 \\
\star & \bar{\Omega}_{22} & 0 & 0 & 0 & 0 & 0 \\
\star & \star & \bar{\Omega}_{33} & 0 & 0 & 0 & 0 \\
\star & \star & \star & \bar{\Omega}_{44} & 0 & 0 & 0 \\
\star & \star & \star & \star & \bar{\Omega}_{55} & 0 & 0 \\
\star & \star & \star & \star & \star & \bar{\Omega}_{66} & 0 \\
\star & \star & \star & \star & \star & \star & \bar{\Omega}_{77}
\end{array}\right]<0,
$$

where, $\quad \bar{\Omega}_{11}=2\left(\mathscr{I} \otimes Q_{1} \mathscr{A}\right)+m_{1}\left(\mathscr{I} \otimes Q_{2}\right)+m_{2}\left(\mathscr{I} \otimes Q_{3}\right)+\left(\mathscr{I} \otimes Q_{6}\right)+\left(\mathscr{I} \otimes \mathscr{M}^{T} \mathscr{M}\right)+\Phi_{1}\left(\mathscr{I} \otimes Q_{1} \mathscr{B}\right)$

$$
\begin{aligned}
& \times\left(\mathscr{I} \otimes \mathcal{Q}_{1} \mathscr{B}\right)^{T}+\omega_{2}\left(\mathscr{I} \otimes Q_{1} \mathscr{C}\right)\left(\mathscr{I} \otimes \mathscr{Q}_{1} \mathscr{C}\right)^{T}+\omega_{4}(\mathscr{I} \otimes \mathscr{H})(\mathscr{I} \otimes \mathscr{H})^{T}-\omega_{5}(\mathscr{I} \otimes \mathscr{H} \mathscr{B})(\mathscr{I} \otimes \mathscr{H} \mathscr{B})^{T} \\
& -\omega_{6}(\mathscr{I} \otimes \mathscr{H} \mathscr{C})(\mathscr{I} \otimes \mathscr{H} \mathscr{C})^{T}+\omega_{8}^{-1}-2(\mathscr{I} \otimes \mathscr{H} \mathscr{A})+2\left(\mathscr{L} \otimes Q_{1} \mathscr{J}\right)-2(\mathscr{L} \otimes \mathscr{H} \mathscr{J})-\omega_{7}^{-1} \text {, } \\
& \bar{\Omega}_{22}=-(1-\lambda)\left(\mathscr{I} \otimes Q_{6}\right) \text {, } \\
& \bar{\Omega}_{33}=-m_{1}\left(\mathscr{I} \otimes Q_{2}\right)+\left(\mathscr{I} \otimes Q_{4}\right) \text {, } \\
& \bar{\Omega}_{44}=-m_{2}\left(\mathscr{I} \otimes Q_{3}\right)-\left(\mathscr{I} \otimes Q_{4}\right) \text {, } \\
& \bar{\Omega}_{55}=-2 \omega(\mathscr{I} \otimes \mathscr{H})+\omega_{8} \omega^{2}(\mathscr{I} \otimes \mathscr{H} \mathscr{A})(\mathscr{I} \otimes \mathscr{H} \mathscr{A})^{T}+\omega_{9} \omega^{2}(\mathscr{I} \otimes \mathscr{H} \mathscr{B})(\mathscr{I} \otimes \mathscr{H} \mathscr{B})^{T}+\omega_{4}^{-1} \\
& +\omega_{3} \omega^{2}(\mathscr{I} \otimes \mathscr{H} \mathscr{C})(\mathscr{I} \otimes \mathscr{H} \mathscr{C})^{T}-\omega_{7} \omega^{2}(\mathscr{L} \otimes \mathscr{H} \mathscr{J})(\mathscr{L} \otimes \mathscr{H} \mathscr{J})^{T}, \\
& \bar{\Omega}_{66}=-1+\left(\mathscr{I} \otimes Q_{5}\right)+\omega_{1}^{-1}-{\omega_{5}^{-1}}^{-1}{\omega_{9}^{-1}}^{-} \\
& \bar{\Omega}_{77}=-(1-\lambda)\left(\mathscr{I} \otimes Q_{5}\right)+\omega_{2}^{-1}-\omega_{6}^{-1}+\oplus_{3}^{-1} \text {. }
\end{aligned}
$$


Remark 9. In [26], the authors addressed the distributed delay, which was a constant value for $m=1$. However in this study, we have taken the distributed time delays of nonlinear multi-agent systems into account and studied the dynamic behaviors. Hence, the constructed multiagent system (5) is more advanced than the current works available in the literature [26]. In the sense of creativity, therefore, our research work is distinct from the previous ones.

Remark 10. In order to portray how to design the control gains to achieve the asymptotic synchronization goal for the considered nonlinear MASs, we take Theorem 2 as an example to design with the following steps (Algorithm 1)

\section{Numerical Example}

Examples of the simulation are provided in this section to highlight the validity of our findings concerning the nonlinear multiagent systems (MASs).

Example 1. Consider the MASs consisting of five followers as follows.

$$
\begin{aligned}
\dot{\mathscr{P}}_{i}(t)= & \mathscr{A} \mathscr{P}_{i}(t)+\mathscr{B} \widetilde{g}\left(\mathscr{P}_{i}(t)\right)+\mathscr{C} \widetilde{g}\left(\mathscr{P}_{i}(t-m(t))\right) \\
& +\mathscr{D} \int_{t-\varrho(t)}^{t} \widetilde{g}\left(\mathscr{P}_{i}(\sigma)\right) \mathrm{d} \sigma-\mathscr{L} \mathscr{P}_{i}(t) .
\end{aligned}
$$

It is assumed that the nonlinear function is taken as $\tilde{g}\left(\mathscr{P}_{i}(t)\right)=0.5 \tan \left(\mathscr{P}_{i}(t)\right), \quad m(t)=1.2 \sin (t)+0.1, \quad m_{1}=$ $0.1, m_{2}=1.3, \lambda=1.2, \varrho(t)=2.2 \sin (t)+0.1$, and $\varrho=2.2$

The system matrices are given as follows:

$$
\begin{aligned}
& \mathscr{A}=\left[\begin{array}{cc}
3.27 & 2.47399 \\
1.51 & 4.7
\end{array}\right] \text {, } \\
& \mathscr{B}=\left[\begin{array}{ll}
1.42 & 2.53 \\
1.41 & 4.52
\end{array}\right] \text {, } \\
& \mathscr{C}=\left[\begin{array}{ll}
2.71 & 1.62 \\
1.48 & 6.32
\end{array}\right] \text {, } \\
& \mathscr{D}=\left[\begin{array}{ll}
2.23 & 3.19 \\
6.22 & 5.57
\end{array}\right] \text {, } \\
& \mathscr{L}=\left[\begin{array}{ccccc}
1 & -1 & 0 & 0 & 0 \\
0 & 1 & -1 & -1 & 0 \\
-1 & 0 & 2 & 0 & 0 \\
0 & 0 & 0 & 1 & -1 \\
0 & 0 & -1 & 0 & 1
\end{array}\right] .
\end{aligned}
$$

Let us select $\omega_{1}=0.4, \omega_{2}=0.5, \omega_{3}=0.9, \omega_{4}=0.5$, $\varpi_{5}=0.4, \omega_{6}=0.1, \oplus_{7}=0.2, \oplus_{8}=0.1, \omega_{9}=0.5, \varpi_{10}=0.5$, $\omega_{11}=0.2$, and $\omega=0.5$. With the support of MATLAB LMI control toolbox, using these values to solving LMI (16) in Theorem 2, a feasible solution is given by

$$
\begin{aligned}
& Q_{1}=\left[\begin{array}{ll}
2.1295 & 3.4059 \\
3.4059 & 7.0172
\end{array}\right], \\
& Q_{2}=\left[\begin{array}{ll}
1.2401 & 0.0213 \\
0.0213 & 0.0102
\end{array}\right], \\
& Q_{3}=\left[\begin{array}{ll}
0.7132 & 0.6265 \\
0.6265 & 0.1835
\end{array}\right], \\
& Q_{4}=\left[\begin{array}{ll}
2.6784 & 0.3216 \\
0.3216 & 1.7563
\end{array}\right], \\
& Q_{5}=\left[\begin{array}{ll}
11.2743 & 0.1059 \\
0.1059 & 9.3452
\end{array}\right], \\
& Q_{6}=\left[\begin{array}{ll}
7.8473 & 6.9345 \\
6.9345 & 4.9624
\end{array}\right], \\
& Q_{7}=\left[\begin{array}{ll}
14.9127 & 0.2353 \\
0.2353 & 11.7654
\end{array}\right] \text {, } \\
& Q_{8}=\left[\begin{array}{ll}
3.8462 & 0.3126 \\
0.3126 & 3.2346
\end{array}\right], \\
& \mathscr{H}=\left[\begin{array}{ll}
3.4942 & 0.0050 \\
0.0050 & 7.9999
\end{array}\right]
\end{aligned}
$$

In this case, an agent's initial conditions are selected as $\mathscr{P}_{1}(0)=[0.23,-0.16]^{T}, \mathscr{P}_{2}(0)=[0.2,-0.2]^{T}, \mathscr{P}_{3}(0)=$ $[0.14,-0.12]^{T}, \mathscr{P}_{4}(0)=[0.1,0.4]^{T}$, and $\mathscr{P}_{5}(0)=[0.18$, $0.24]^{T}$, and the agent's equilibrium point is plotted in Figure 1. Then, the stability of the multiagent system is shown in Figure 2. It can be shown that all followers will enter the region around the leaderless asymptotically, confirming the validity of Theorem 2 .

Example 2. Consider the leader system (40) and controller of the follower system (47) as follows.

$$
\begin{aligned}
\dot{\mathcal{S}}_{i}(t)= & \mathscr{A} \mathcal{S}_{i}(t)+\mathscr{B} \tilde{g}\left(\mathcal{S}_{i}(t)\right)+\mathscr{C} \tilde{g}\left(\mathcal{S}_{i}(t-m(t))\right) \\
& +\mathscr{D} \int_{t-\varrho(t)}^{t} \tilde{g}\left(\mathcal{S}_{i}(\sigma)\right) \mathrm{d} \sigma+\mathscr{K}_{i}(t) .
\end{aligned}
$$

It is assumed that the nonlinear function is taken as $\tilde{g}\left(\mathcal{S}_{i}(t)\right)=0.5 \tan \left(\mathcal{S}_{i}(t)\right), \quad m(t)=1.8 \sin (t)+0.2, \quad m_{1}=$ $0.2, m_{2}=2.0, \lambda=1.8, \varrho(t)=1.4 \sin (t)+0.1$, and $\varrho=1.4$. The system matrices are 
Algorithm

Step 1. Initialize the system parameters $\mathscr{A}, \mathscr{B}, \mathscr{C}$, and $\mathscr{D}$ and some positive constants $m_{1}, m_{2}, \varrho$, and $\lambda$

Step 2. Select an appropriate matrix $\mathscr{M}$ according to Assumption 1

Step 3. Choose the control gain matrix $\mathscr{J}$

Step 4 . There exists positive scalars $\omega, \emptyset_{\mathfrak{K}},(\Re=1, \ldots, 12)$ symmetric matrices $\mathbb{Q}_{\mathfrak{K}},(\mathfrak{K}=1, \ldots, 8)>0$, any matrix $\mathscr{H}$, and LMI (52) hold If success, the procedure further moves to next step

Otherwise, the procedure turns back to adjust the system parameters and control gain matrix in Step 1 and Step 3

Step 5. Based on the proper control gain matrix, we design a linear feedback control

Algorithm 1: Algorithm to design the linear feedback control.

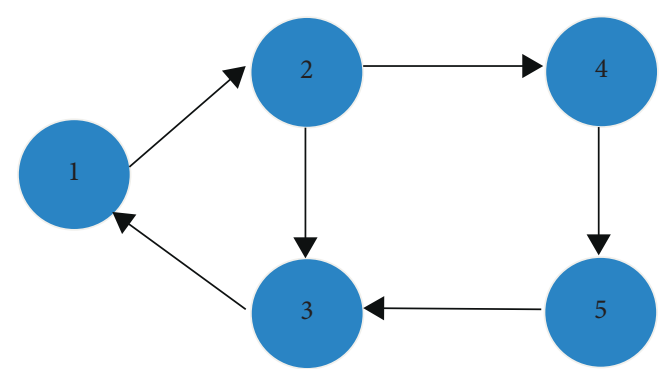

Figure 1: Communication of MASs without leader.

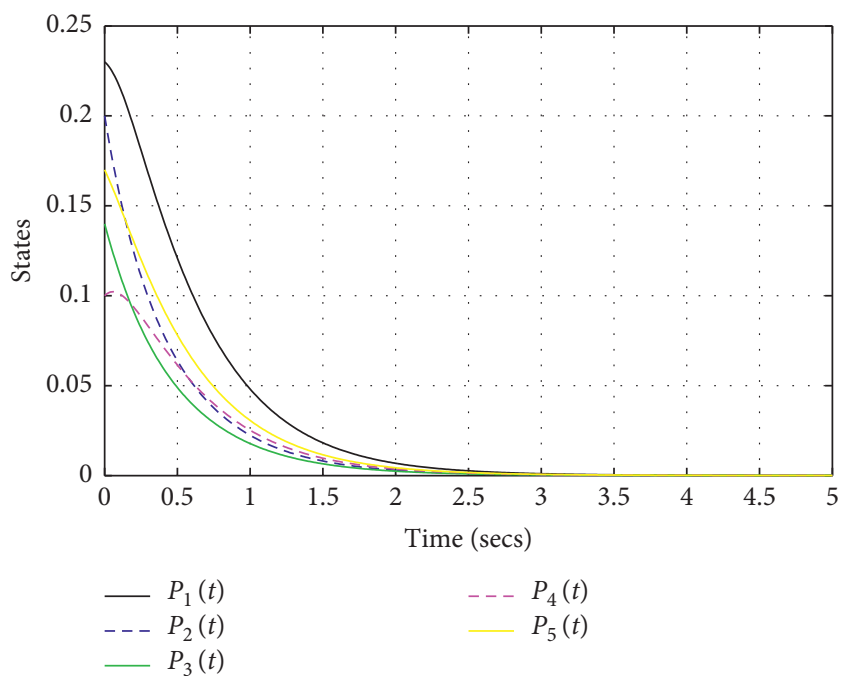

FIgure 2: The stability of MASs in Theorem 2.

$$
\begin{aligned}
& \mathscr{A}=\left[\begin{array}{cc}
3.12 & 0.1671 \\
0.01 & 2.00
\end{array}\right], \\
& \mathscr{B}=\left[\begin{array}{ll}
2.42 & 0.23 \\
0.53 & 3.52
\end{array}\right], \\
& \mathscr{C}=\left[\begin{array}{ll}
3.71 & 3.21 \\
1.43 & 1.32
\end{array}\right], \\
& \mathscr{D}=\left[\begin{array}{ll}
2.23 & 3.43 \\
1.54 & 5.57
\end{array}\right] .
\end{aligned}
$$

The Laplacian matrix $\mathscr{L}$ and the corresponding control gain matrix $\mathscr{J}$ shall be calculated as
TABLE 1: Comparison table of lower and upper bounds of Theorem 1.

\begin{tabular}{lccccc}
\hline Theorem 2 & $m_{1}$ & $m_{2}$ & $\lambda$ & $\varrho$ & Solution \\
1 & 0.2 & 1.8 & 1.8 & 1.4 & Feasible \\
2 & 0.2 & 2.0 & 1.8 & 1.4 & Feasible \\
3 & 0.2 & 2.5 & 1.8 & 1.4 & Infeasible \\
\hline
\end{tabular}

$$
\begin{aligned}
& \mathscr{L}=\left[\begin{array}{ccccc}
1 & -1 & 0 & 0 & 0 \\
0 & 1 & -1 & -1 & -1 \\
-1 & 0 & 2 & 0 & 0 \\
0 & 0 & 0 & 1 & -1 \\
0 & 0 & -1 & 0 & 1
\end{array}\right], \\
& \mathscr{J}=\left[\begin{array}{ccc}
0.000004 & 0.000001 \\
0.000001 & 0.00004
\end{array}\right] .
\end{aligned}
$$

Let us select $\Phi_{1}=0.08, \Phi_{2}=0.06, \omega_{3}=0.04, \Phi_{4}=0.07$, $\omega_{5}=0.03, \omega_{6}=0.12, \omega_{7}=0.05, \omega_{8}=0.03, \omega_{9}=0.02$, $\omega_{10}=0.06, \omega_{11}=0 ; 04, \omega_{12}=0 ; 062$, and $\omega=0.001$. With the support of MATLAB LMI control toolbox, using these values and by solving LMI (28) in Theorem 2, the following feasible solutions are obtained as

$$
\begin{aligned}
& \mathscr{Q}_{1}=\left[\begin{array}{ll}
2.8877 & 0.0000 \\
0.0000 & 0.0172
\end{array}\right], \\
& \mathscr{Q}_{2}=\left[\begin{array}{ll}
4.6501 & 0.0000 \\
0.0000 & 3.1002
\end{array}\right], \\
& Q_{3}=\left[\begin{array}{ll}
1.0006 & 0.0000 \\
0.0000 & 2.6003
\end{array}\right], \\
& \mathscr{Q}_{4}=\left[\begin{array}{ll}
0.1008 & 0.0000 \\
0.0000 & 0.1002
\end{array}\right], \\
& \mathscr{Q}_{5}=\left[\begin{array}{ll}
11.2743 & 0.0000 \\
0.0000 & 9.3452
\end{array}\right], \\
& \mathscr{Q}_{6}=\left[\begin{array}{ll}
7.3567 & 0.0000 \\
0.0000 & 2.4904
\end{array}\right], \\
& \mathscr{Q}_{7}=\left[\begin{array}{ll}
8.9127 & 0.0000 \\
0.0000 & 9.5272
\end{array}\right], \\
& \mathscr{Q}_{8}=\left[\begin{array}{ll}
0.3657 & 0.0000 \\
0.0000 & 6.8505
\end{array}\right], \\
& \mathscr{H}=\left[\begin{array}{ll}
0.4941 & 0.0000 \\
0.0000 & 0.9999
\end{array}\right] .
\end{aligned}
$$






Figure 3: Communication of MASs with leader.

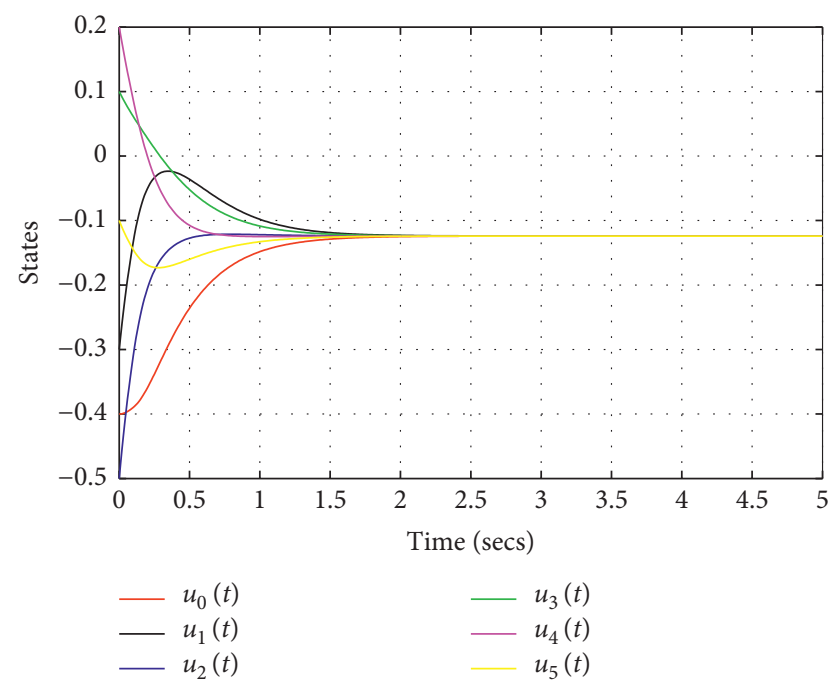

FIgURE 4: State trajectories of MASs.

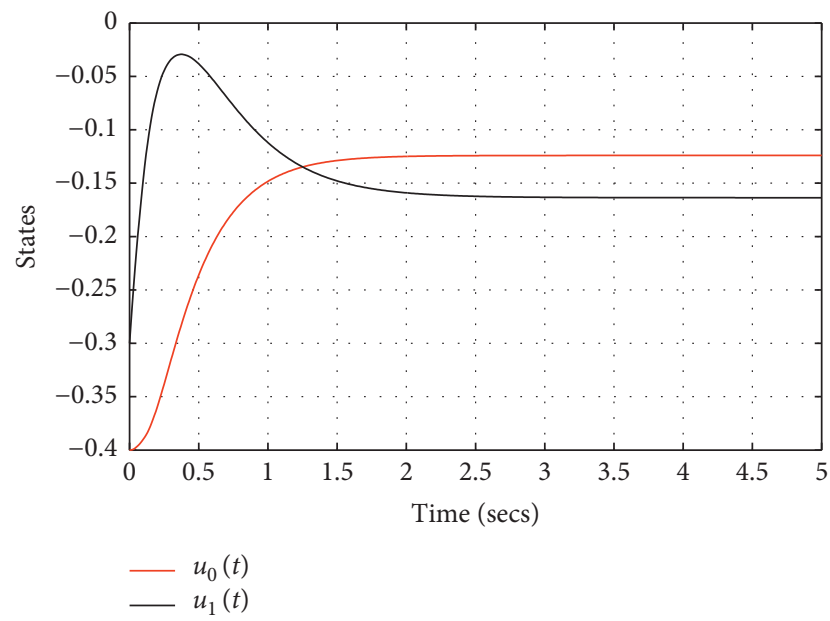

FIGURE 5: Synchronization path states $\mathscr{U}_{1}(t)$ vs. $\mathscr{U}_{0}(t)$ and its error $\mathcal{S}_{1}(t)$ without any control inputs.

In this case, an agent's initial conditions are selected as Table 1: $\mathscr{U}_{0}(0)=[-0.4,0.7]^{T}, \mathscr{U}_{1}(0)=[-0.3,-0.6]^{T}$, $\mathscr{U}_{2}(0)=[-0.5,-0.2]^{T}, \mathscr{U}_{3}(0)=[0.1,-0.3]^{T}, \mathscr{U}_{4}(0)=$ $[0.2,0.4]^{T}$, and $\mathscr{U}_{5}(0)=[-0.1,0.4]^{T}$, and the agent's synchronization is shown in Figure 3. It can be observed that all followers will enter the region around the leader asymptotically, confirming the validity of Theorem 2 . The state trajectories of

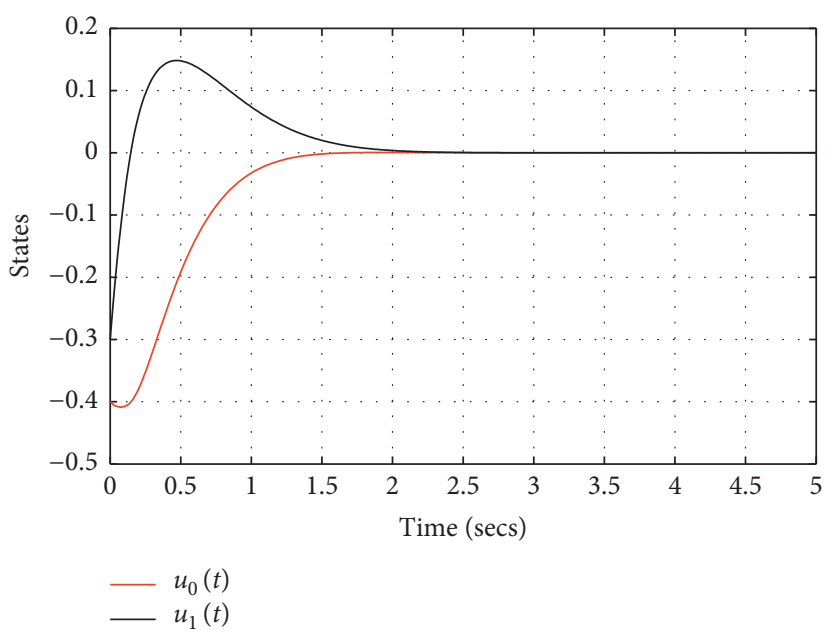

FIGURE 6: Synchronization path states $\mathscr{U}_{1}(t)$ vs. $\mathscr{U}_{0}(t)$ and its error $\mathcal{S}_{1}(t)$ with control inputs.

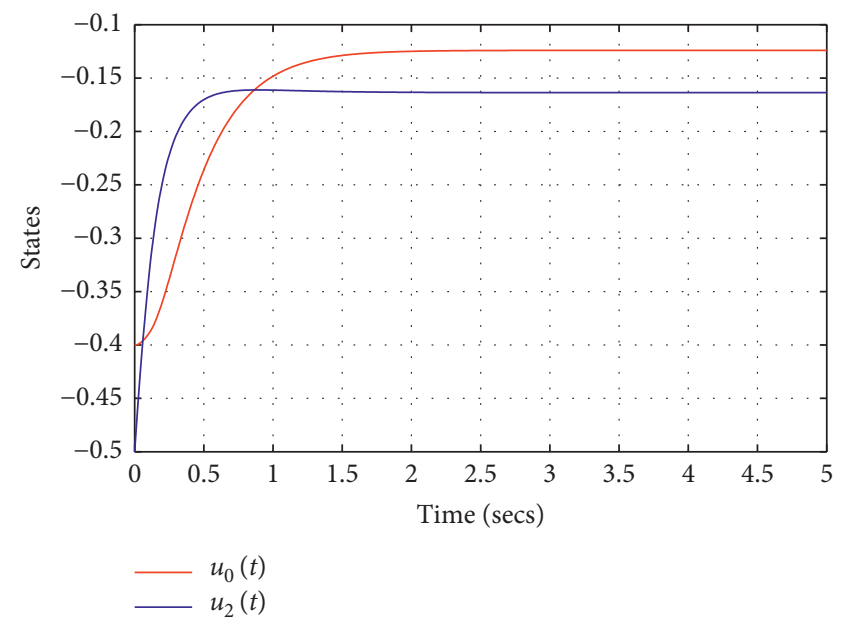

FIgURE 7: Synchronization path states $\mathscr{U}_{2}(t)$ vs. $\mathscr{U}_{0}(t)$ and its error $\mathcal{S}_{2}(t)$ without any control inputs.

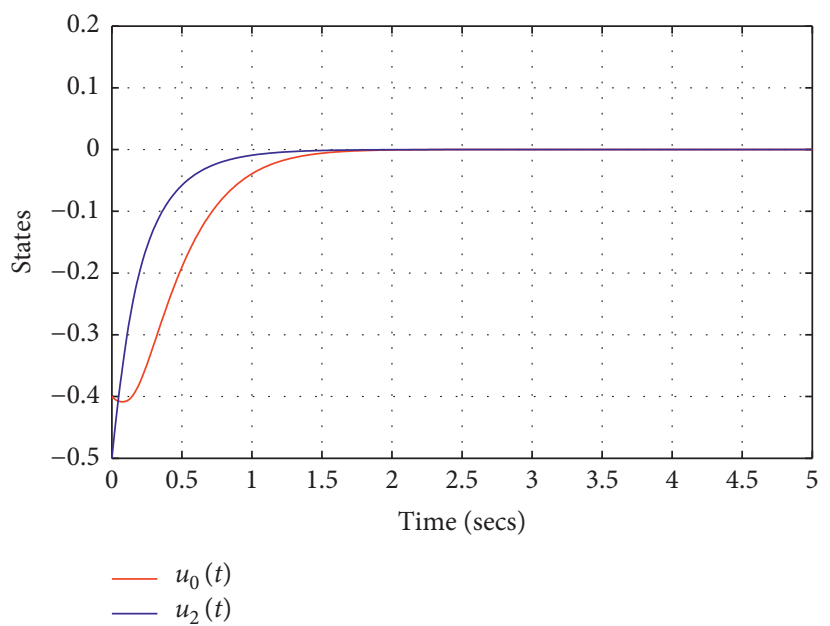

FIgURE 8: Synchronization path states $\mathscr{U}_{2}(t)$ vs. $\mathscr{U}_{0}(t)$ and its error $\mathcal{S}_{2}(t)$ with control inputs. 


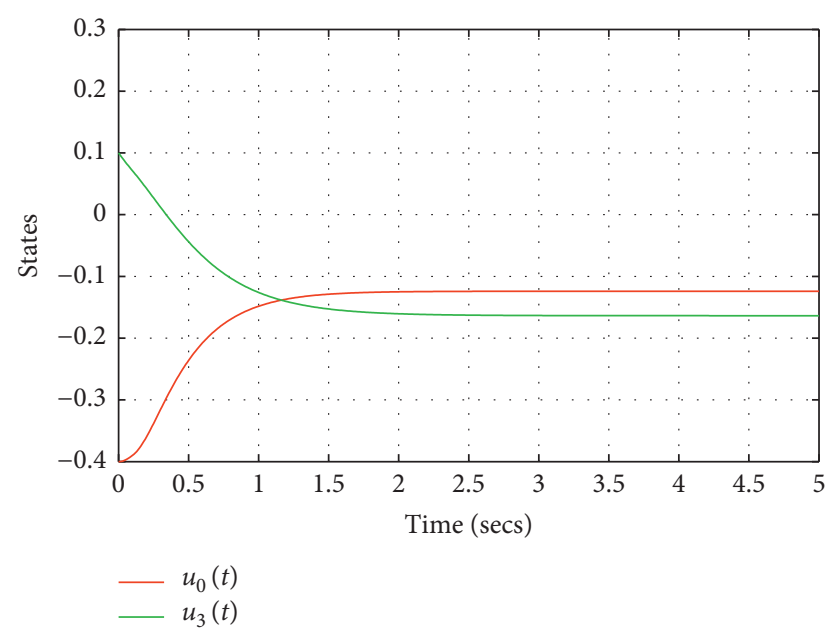

FIGURE 9: Synchronization path states $\mathscr{U}_{3}(t)$ vs. $\mathscr{U}_{0}(t)$ and its error $\mathcal{S}_{3}(t)$ without any control inputs.

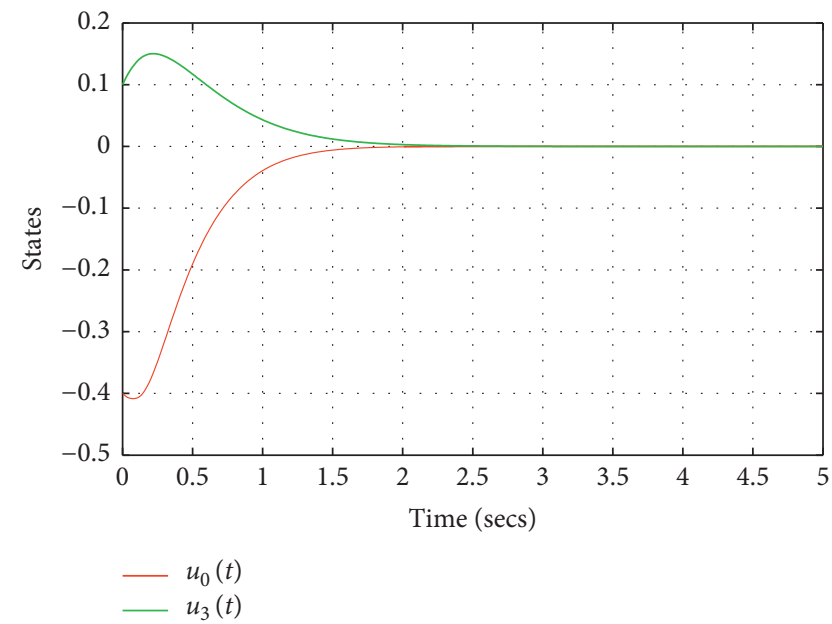

FIGURE 10: Synchronization path states $\mathscr{U}_{3}(t)$ vs. $\mathscr{U}_{0}(t)$ and its error $\mathcal{S}_{3}(t)$ with control inputs.

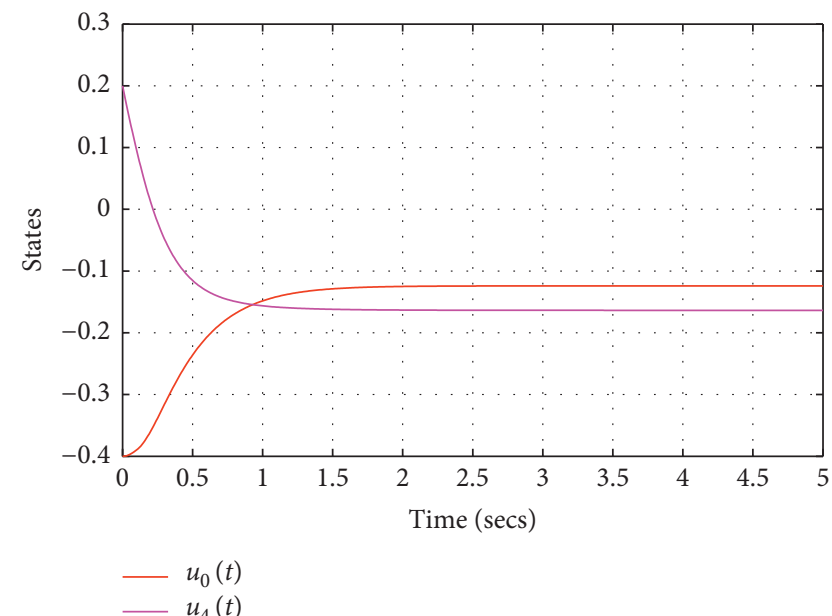

FIgURE 11: Synchronization path states $\mathscr{U}_{4}(t)$ vs. $\mathscr{U}_{0}(t)$ and its error $\mathcal{S}_{4}(t)$ without any control inputs.

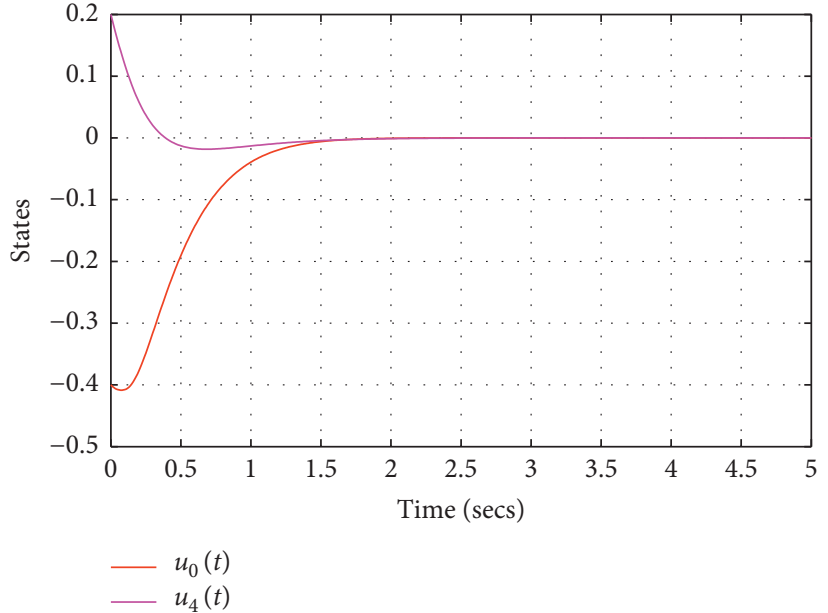

FIgURE 12: Synchronization path states $\mathscr{U}_{4}(t)$ vs. $\mathscr{U}_{0}(t)$ and its error $\mathcal{S}_{4}(t)$ with control inputs.

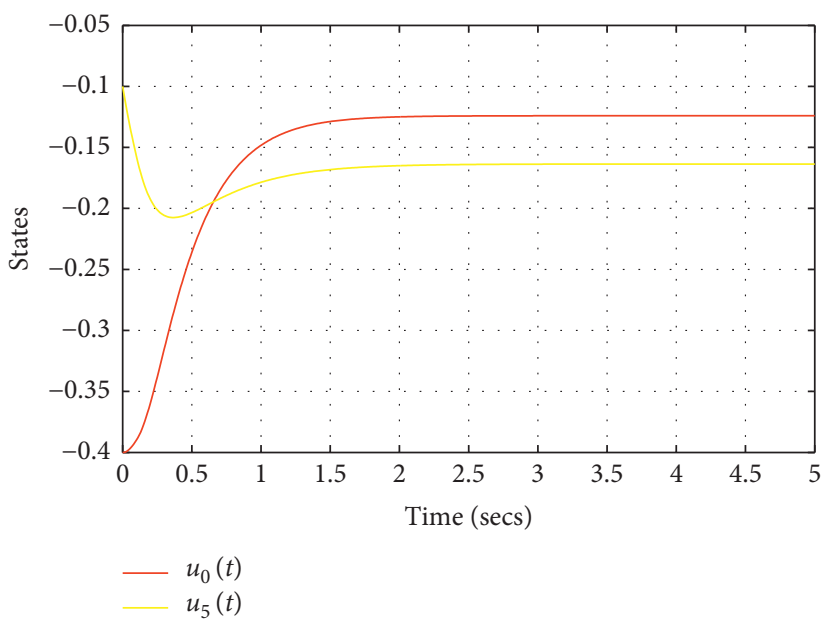

FIgURE 13: Synchronization path states $\mathscr{U}_{5}(t)$ vs. $\mathscr{U}_{0}(t)$ and its error $\delta_{5}(t)$ without any control inputs.

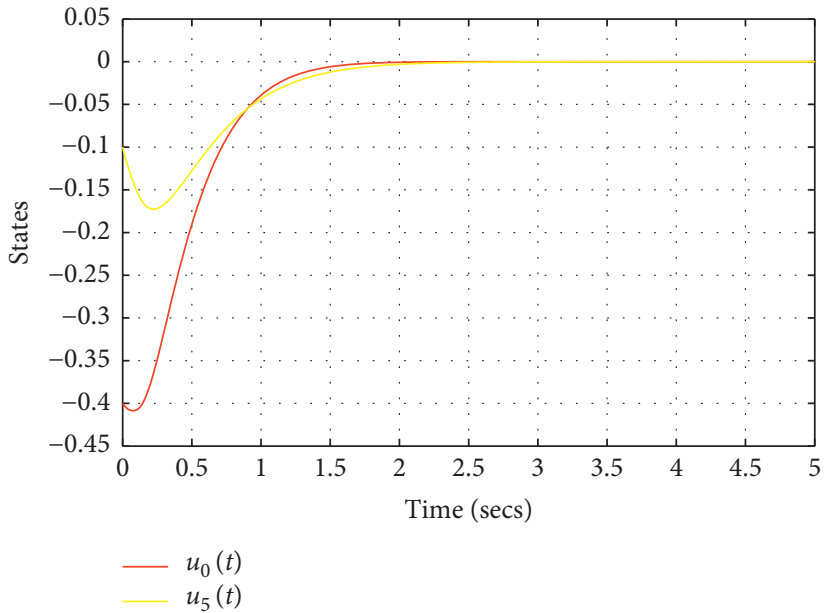

FIgURE 14: Synchronization path states $\mathscr{U}_{5}(t)$ vs. $\mathscr{U}_{0}(t)$ and its error $\mathcal{S}_{5}(t)$ with control inputs. 
MASs are shown in Figure 4. Then, the synchronization of the multi-agent systems without and with controllers is shown in Figures 5-14, respectively. The feasibility of the theorem 2 has been represented in the Table 1.

\section{Conclusion}

In this research work, we have studied the asymptotic stability and synchronization of nonlinear multi-agent systems with mixed delays. The asymptotic stability criterion for solving a suitable Lyapunov-Krasovskii functional by using the LMI condition in a nonlinear multi-agent systems was utilized. Moreover, we have proposed a novel linear feedback control strategy in order to get the less conservativeness. Also, a leader-follower problem has been solved by constructing an appropriate Lyapunov-Krasovskii functional and used the LMI condition into the multiagent system and then attained the asymptotic synchronization. Finally, two numerical examples with simulations were presented to demonstrate and justify the main proofs derived in the theoretical section.

\section{Data Availability}

No data were used to support the findings of this study.

\section{Conflicts of Interest}

The authors declare that they have no conflicts of interest.

\section{Acknowledgments}

The article has been written with the joint partial financial support of the RUSA-Phase 2.0 grant sanctioned vide letter no. F 24-51/2014-U, Policy (TN Multi-Gen), Department of Education, Government of India, UGC-SAP (DRS-I) vide letter no. F.510/8/DRS-I/2016 (SAP-I), and DST (FISTPhase I) vide letter no. SR/FIST/MS-I/2018-17, the National Science Centre in Poland (DEC-2017/25/B/ST7/02888) and J. Alzabut would like to thank Prince Sultan University for supporting this work.

\section{References}

[1] R. W. Beard, T. W. McLain, M. A. Goodrich, and E. P. Anderson, "Coordinated target assignment and intercept for unmanned air vehicles," IEEE Transactions on Robotics and Automation, vol. 18, no. 6, pp. 911-922, 2002.

[2] J. A. Fax and R. M. Murray, "Information flow and cooperative control of vehicle formations," IEEE Transactions on Automatic Control, vol. 49, no. 9, pp. 1465-1476, 2004.

[3] W. Ren and N. Sorensen, "Distributed coordination architecture for multi-robot formation control," Robotics and Autonomous Systems, vol. 56, no. 4, pp. 324-333, 2008.

[4] J. H. Holland, Adaptation in Natural and Artificial Systems, The MIT Press, Cambridge, MA, USA, 2nd edition, 1992.

[5] G. Agha, Actors: A Model of Concurrent Computation in Distributed Systems, The MIT Press, Cambridge, MA, USA, 1986.
[6] J. Hu, J. Cao, K. Yuan, and T. Hayat, "Cooperative tracking for nonlinear multi-agent systems with hybrid time-delayed protocol," Neurocomputing, vol. 171, pp. 171-178, 2016.

[7] B. Zhou, X. Liao, T. Huang, and G. Chen, "Leader-following exponential consensus of general linear multi-agent systems via event-triggered control with combinational measurements," Applied Mathematics Letters, vol. 40, no. 2, pp. 35-39, 2015.

[8] H. Liang, G. Liu, H. Zhang, and T. Huang, "Neural-networkbased event-triggered adaptive control of nonaffine nonlinear multiagent systems with dynamic uncertainties," IEEE Transactions on Neural Networks and Learning Systems, p. 1, 2020.

[9] M.-J. Park, S.-H. Lee, O.-M. Kwon, J. H. Park, and S.-G. Choi, "Betweenness centrality-based consensus protocol for second-order multiagent systems with sampled-data," IEEE Transactions on Cybernetics, vol. 47, no. 8, pp. 2067-2078, 2017.

[10] M.-J. Park, O.-M. Kwon, and A. Seuret, "Weighted consensus protocols design based on network centrality for multi-agent systems with sampled-data," IEEE Transactions on Automatic Control, vol. 62, no. 6, pp. 2916-2922, 2017.

[11] C. Wang, Z. Zuo, Z. Lin, and Z. Ding, "Consensus control of a class of Lipschitz nonlinear systems with input delay," IEEE Transactions on Circuits and Systems I: Regular Papers, vol. 62, no. 11, pp. 2730-2738, 2015.

[12] Y. Wang, J. Cao, H. Wang, A. Alsaedi, and F. U. Alsaadi, "Exponential consensus for nonlinear multi-agent system with communication and input delays via hybrid controller," Asian Journal of Control, vol. 20, no. 5, pp. 1-12, 2018.

[13] O. M. Kwon, M. J. Park, J. H. Park, S. M. Lee, and E. J. Cha, "On stability analysis for neural networks with interval timevarying delays via some new augmented lyapunov-krasovskii functional," Communications in Nonlinear Science and Numerical Simulation, vol. 19, no. 9, pp. 3184-3201, 2014.

[14] W. Qin, Z. Liu, and Z. Chen, "Impulsive observer-based consensus control for multi-agent systems with time delay," International Journal of Control, vol. 88, no. 9, pp. 1789-1804, 2015.

[15] S. Rasoolinasab, S. Mobayen, A. Fekih, P. Narayan, and Y. Yao, "A composite feedback approach to stabilize nonholonomic systems with time varying time delays and nonlinear disturbances," ISA Transactions, vol. 101, pp. 177$188,2020$.

[16] S. Mobayen, F. Bayat, H. Omidvar, and A. Fekih, "Robust global controller design for discrete-time descriptor systems with multiple time-varying delays," International Journal of Robust and Nonlinear Control, vol. 30, no. 7, pp. 2809-2831, 2020.

[17] R. Song, B. Wang, and Q. Zhu, "Delay-dependent stability of nonlinear hybrid neutral stochastic differential equations with multiple delays," International Journal of Robust and Nonlinear Control, vol. 31, no. 1, pp. 250-267, 2021.

[18] M. J. Park, S. H. Lee, O. M. Kwon, and A. Seuret, "Closedcentrality based synchronization criteria for complex dynamical networks with interval time-varying coupling delays," IEEE Transactions on Cybernetics, vol. 48, no. 7, pp. 2018-2202, 2018.

[19] J. Hu, J. Yu, and J. Cao, "Distributed containment control for nonlinear multi-agent systems with time-delayed protocol," Asian Journal of Control, vol. 18, no. 2, pp. 747-756, 2016.

[20] Q. Jia, W. K. S. Tang, and W. A. Halang, "Leader following of nonlinear agents with switching connective network and 
coupling delay," IEEE Transactions on Circuits and Systems I: Regular Papers, vol. 58, no. 10, pp. 2508-2519, 2011.

[21] W. Ni and D. Cheng, "Leader-following consensus of multiagent systems under fixed and switching topologies," Systems and Control Letters, vol. 59, no. 3-4, pp. 209-217, 2010.

[22] R. Rakkiyappan, B. Kaviarasan, and J. Cao, "Leader-following consensus of multi-agent systems via sampled-data control with randomly missing data," Neurocomputing, vol. 161, pp. 132-147, 2015.

[23] K. Subramanian, P. Muthukumar, and Y. Hoon Joo, "Leaderfollowing consensus of nonlinear multi-agent systems via reliable control with time-varying communication delay," International Journal of Control, Automation and Systems, vol. 17, pp. 1-9, 2019.

[24] Z. Meng, W. Ren, Y. Cao, and Z. You, "Leaderless and leaderfollowing consensus with communication and input delays under a directed network topology," IEEE Transactions on Systems, Man, and Cybernetics, Part B (Cybernetics), vol. 41, no. 1, pp. 75-88, 2011.

[25] W. He, B. Zhang, Q. L. Han, F. Qian, J. Kurths, and J. Cao, "Leader-Following consensus of nonlinear multiagent systems with stochastic sampling," IEEE Transactions on Cybernetics, vol. 47, no. 2, pp. 327-338, 2017.

[26] H. Cha, "Leader-following consensus of nonlinear multiagent systems with mixed delays and uncertain parameters via adaptive pinning intermittent control," Nonlinear Analysis: Hybrid Systems, vol. 22, pp. 202-214, 2016.

[27] A. Pratap, R. Raja, R. P. Agarwal, and J. Cao, "Stability analysis and robust synchronization of fractional-order competitive neural networks with different time scales and impulsive perturbations," International Journal of Adaptive Control and Signal Processing, vol. 33, no. 11, pp. 1635-1660, 2019.

[28] K. Balasundaram, R. Raja, A. Pratap, and S. Chandrasekaran, "Impulsive effects on competitive neural networks with mixed delays: existence and exponential stability analysis," Mathematics and Computers in Simulation, vol. 155, pp. 290-302, 2019.

[29] R. Senthil, R. Raja, R. Samidurai, J. Cao, and X. Li, "Passivity analysis of uncertain stochastic neural network with leakage and distributed delays under impulsive perturbations," Kybernetika, vol. 54, no. 1, pp. 3-29, 2018.

[30] F. Kong, Q. Zhu, and T. Huang, "New fixed-time stability lemmas and applications to the discontinuous fuzzy inertial neural networks," IEEE Transactions on Fuzzy Systems, 2020.

[31] W. Li, H. Zhou, Z.-W. Liu, Y. Qin, and Z. Wang, "Impulsive coordination of nonlinear multi-agent systems with multiple leaders and stochastic disturbance," Neurocomputing, vol. 171, pp. 73-81, 2016.

[32] M. S. Ali, M. Usha, Q. X. Zhu, and S. Shanmugam, "Synchronization analysis for stochastic T-S fuzzy complex networks with Markovian jumping parameters and mixed time-varying delays via impulsive control," Mathamatical Problem in Engineering, vol. 2020, p. 27, 2020.

[33] F. Kong, Q. Zhu, R. Sakthivel, and A. Mohammadzadeh, "Fixed-time synchronization analysis for discontinuous fuzzy inertial neural networks with parameter uncertainties," Neurocomputing, vol. 422, pp. 295-313, 2021.

[34] F. Kong and Q. Zhu, "New fixed-time synchronization control of discontinuous inertial neural networks via indefinite Lyapunov-Krasovskii functional method," International Journal of Robust and Nonlinear Control, vol. 31, no. 2, pp. 471-495, 2021.

[35] Z. Li, Z. Duan, G. Chen, and L. Huang, "Consensus of multiagent systems and synchronization of complex networks: a unified viewpoint," IEEE Transactions on Circuits and Systems I: Regular Papers, vol. 57, no. 1, pp. 213-224, 2010.

[36] H. Su, Z Rong, M. Z. Chen, X. Wang, G. Chen, and H. Wang, "Decentralized adaptive pinning control for cluster synchronization of complex dynamical networks," IEEE Transactions on Cybernetics, vol. 43, no. 1, pp. 394-9, 2013.

[37] A. Pratap, R. Raja, C. Sowmiya, O. Bagdasar, J. Cao, and G. Rajchakit, "Global projective lag synchronization of fractional order memristor based BAM neural networks with mixed time varying delays," Asian Journal of Control, vol. 22, no. 1, pp. 570-583, 2020.

[38] A. Pratap, R. Raja, J. Alzabut, J. Cao, G. Rajchakit, and C. Huang, "Mittag-leffler stability and adaptive impulsive synchronization of fractional order neural networks in quaternion field," Mathematical Methods in the Applied Sciences, vol. 43, no. 10, pp. 6223-6253, 2020.

[39] Z. Guan, Z. Liu, G. Feng et al., "Synchronization of complex dynamical networks with time-varying delays via impulsive distributed control," IEEE Transactions on Circuits and Systems I: Regular Papers, vol. 57, no. 8, pp. 2182-2195, 2010.

[40] G. Rajchakit, P. Chanthorn, M. Niezabitowski, R. Raja, D. Baleanu, and A. Pratap, "Impulsive effects on stability and passivity analysis of memristor-based fractional-order competitive neural networks," Neurocomputing, vol. 417, pp. 290-301, 2010.

[41] C. Hu and H. Jiang, "Pinning synchronization for directed networks with node balance via adaptive intermittent control," Nonlinear Dynamics, vol. 80, no. 1-2, pp. 295-307, 2015.

[42] Y. Wang, J. Cao, and J. Hu, "Pinning consensus for multiagent systems with non-linear dynamics and time-varying delay under directed switching topology," IET Control Theory \& Applications, vol. 8, no. 17, pp. 1931-1939, 2014.

[43] L. Guo, H. Pan, and X. Nian, "Adaptive pinning control of cluster synchronization in complex networks with lurie-type nonlinear dynamics," Neurocomputing, vol. 182, no. 19, pp. 294-303, 2016.

[44] X. He, W. He, J. Shi, and C. Sun, "Boundary vibration control of variable Length Crane systems in two-dimensional space with Output constraints," IEEE/ASME Transactions on Mechatronics, vol. 22, no. 5, pp. 1952-1962, 2017.

[45] T. Saravanakumar, N. H. Muoi, and Q. Zhu, "Finite-time sampled-data control of switched stochastic model with non-deterministic actuator faults and saturation nonlinearity," Journal of the Franklin Institute, vol. 357, no. 18, pp. 13637-13665, 2020.

[46] W. He, T. Meng, X. He, and S. S. Ge, "Unified iterative learning control for flexible structures with input constraints," Automatica, vol. 96, pp. 326-336, 2018.

[47] S. Mobayen and J. Mab, "Robust finite-time composite nonlinear feedback control for synchronization of uncertain chaotic systems with nonlinearity and time-delay," Chaos, Solitons and Fractals, vol. 114, pp. 46-54, 2018.

[48] L. M. Pecora and T. L. Carroll, "Synchronization in chaotic systems," Physical Review Letters, vol. 64, no. 8, pp. 821-824, 1990.

[49] S. Boyd, L. Ghaoui, E. Feron, and V. Balakrishnan, Linear Matrix Inequalities in System and Control Theory, SIAM, Philadelphia, PA, USA, 1994.

[50] Q. Yao, L. Wang, and Y. Wang, "Existence-uniqueness and stability of reaction-diffusion stochastic Hopfield neural networks with S-type distributed time delays," Neurocomputing, vol. 275, pp. 470-477, 2018. 